\title{
COHOMOLOGICAL INDUCTION AND UNIFORM MEASURE EQUIVALENCE
}

\author{
THOMAS GOTFREDSEN AND DAVID KYED
}

\begin{abstract}
We construct a general cohomological induction isomorphism from a uniform measure equivalence of locally compact, second countable, unimodular groups which, as a special case, yields that the graded cohomology algebras of quasi-isometric, connected, simply connected, nilpotent Lie groups are isomorphic. This unifies results of Shalom and Sauer and also provides new insight into the quasi-isometry classification problem for low dimensional nilpotent Lie groups.
\end{abstract}

\section{INTRODUCTION}

Geometric and measurable group theory originated in the the works of Gromov and is by now a well developed and important tool in the study of countable discrete groups; see e.g. Gro84, Fur99a, Fur99b, OW80 and references therein. One of the many insights from the beginning of the present century, mainly due to Shalom, Shalom-Monod MS06] and Sauer Sau06, is that group cohomology, interacts well with one of the fundamental concepts in geometric group theory, namely that of quasi-isometry [Roe03, CdlH16]. These results are mainly concerned with the class of discrete, countable groups, but in recent years, increasing emphasis has been put on the the more general setting of locally compact groups BHI18, BFS13, CLM18, BR18a, KKR17, KKR18, and results concerning the interplay between group cohomology and quasi-isometry are now beginning to emerge in this setting as well [BR18b, SS18. The present article provides a contribution to this line of research by proving that the central results due Shalom, Shalom-Monod and Sauer mentioned above, admit natural generalisations to the class of unimodular, locally compact, second countable groups. Actually, our primary focus will not be on quasi-isometry but rather on the related notion known as (uniform) measure equivalence, which is a measurable analogue of quasi-isometry, originally introduced for discrete groups by Gromov in Gro93. Uniform measure equivalence can be defined also for unimodular, locally compact second countable groups [BFS13, KKR17], and for compactly generated, unimodular groups it was shown in [KKR17] that, just as in the discrete case, uniform measure equivalence implies quasi-isometry and that the two notions coincide when the groups in question are amenable. Our primary focus will therefore be on uniformly measure equivalent topological groups and our first main result, drawing inspiration from [MS06, Proposition 4.6], is the following reciprocity principle in group cohomology; for the basic definitions concerning cohomology and uniform measure equivalence, see Section 2 .

Theorem A. If $G$ and $H$ are uniformly measure equivalent, locally compact, second countable, unimodular groups, then for any uniform measure equivalence coupling $(\Omega, \eta, X, \mu, Y, \nu, i, j)$

2010 Mathematics Subject Classification. 20F65, 22D05, 57M07, 20J06, 57T10 .

Key words and phrases. Locally compact groups, measure equivalence, quasi-isometry, cohomology, nilpotent Lie groups. 
and any Fréchet $G \times H$-module $\mathcal{E}$ there exists an isomorphism of topological vector spaces

$$
\mathrm{H}^{n}\left(G, L_{\mathrm{loc}}^{2}(\Omega, \mathcal{E})^{H}\right) \stackrel{\sim}{\longrightarrow} \mathrm{H}^{n}\left(H, L_{\mathrm{loc}}^{2}(\Omega, \mathcal{E})^{G}\right),
$$

for all $n \geqslant 0$. In particular, when $\mathcal{E}=\mathbb{R}$ with trivial $G \times H$-action, this induces an isomorphism

$$
\mathrm{H}^{n}\left(G, L^{2}(X)\right) \simeq \mathrm{H}^{n}\left(H, L^{2}(Y)\right) .
$$

As a consequence of Theorem $\AA$ we also obtain a generalisation of [Sau06, Theorem 5.1]. For the statement, recall that a group $G$ is said to have (Shalom's) property $H_{T}$ if $\underline{H}^{1}(G, \mathcal{H})=\{0\}$ for every unitary $G$-module $\mathcal{H}$ with no non-trivial fixed-points; here $\underline{H}^{1}(G, \mathcal{H})$ denotes the first reduced cohomology of $G$ with coefficients in $\mathcal{H}$; i.e. the maximal Hausdorff quotient of the ordinary cohomology $\mathrm{H}^{1}(G, \mathcal{H})$ (see Section 2 for more details). In what follows, we will by property $H_{T}^{n}$ mean the obvious extension of property $H_{T}$ to higher cohomologies. Recall also that the total cohomology $\mathrm{H}^{*}(G, \mathbb{R}):=\oplus_{n \geqslant 0} \mathrm{H}^{n}(G, \mathbb{R})$ and its reduced counterpart $\underline{\mathrm{H}}^{*}(G, \mathbb{R}):=\oplus_{n \geqslant 0} \underline{\mathrm{H}}^{n}(G, \mathbb{R})$ become graded, unital $\mathbb{R}$-algebras with respect to the cup product; see Sections 2.1.1 and 2.1.3 for further details on this. Our generalisation of [Sau06, Theorem 5.1] can now be stated as follows:

Theorem B. If $G$ and $H$ are uniformly measure equivalent, locally compact, second countable, unimodular groups satisfying property $H_{T}^{n}$ for all $n \in \mathbb{N}$ then the associated reduced cohomology algebras $\underline{\mathrm{H}}^{*}(G, \mathbb{R})$ and $\underline{\mathrm{H}}^{*}(H, \mathbb{R})$ are isomorphic as graded, unital $\mathbb{R}$-algebras.

As already mentioned, for compactly generated amenable groups, quasi-isometry and uniform measure equivalence coincide, and when the $n$-th Betti number $\beta^{n}(G):=\operatorname{dim}_{\mathbb{R}} \mathrm{H}^{n}(G, \mathbb{R})$ is finite, then $\mathrm{H}^{n}(G, \mathbb{R})$ is automatically Hausdorff [Gui80, III, Prop. 3.1], so that we indeed recover Sauer's result [Sau06, Theorem 5.1]. We remark that the class of connected, simply connected (csc) nilpotent Lie groups (as well as the class of finitely generated, nilpotent groups) satisfies the assumptions in Theorem B and that these furthermore have finite Betti numbers in all degrees; see Section 2.1.1 for details on this. We therefore, in particular, also obtain the following.

Corollary C. If $G$ and $H$ are quasi-isometric, connected, simply connected, nilpotent Lie groups then the cohomology algebras $\mathrm{H}^{*}(G, \mathbb{R})$ and $\mathrm{H}^{*}(H, \mathbb{R})$ are isomorphic as graded unital $\mathbb{R}$-algebras.

For csc nilpotent Lie groups containing lattices, i.e. those admitting a rational structure [Mal51, Mal49, the isomorphisms in Theorem B and Corollary C] were already known to experts in the field, as they can be be deduced from [Sau06, Theorem 5.1] via [Nom54, Theorem 1]. In this way Theorem $B$ and Corollary $C$ provide a more natural approach, covering also csc nilpotent groups without lattices, and, as a special case, it also gives an isomorphism of graded $\mathbb{R}$-algebras $\underline{\mathrm{H}}^{*}(\Gamma, \mathbb{R}) \simeq \underline{\mathrm{H}}^{*}(G, \mathbb{R})$ for any locally compact second countable, unimodular group $G$ with a uniform lattice $\Gamma \leqslant G$; see [KKR18, Proposition 6.11].

As an application of our results, we show in Section 5 how the results above can be used to improve on the quasi-isometry classification programme for csc nilpotent Lie groups of dimension 7. In particular, we show that for the seven 1-parameter families of 7-dimensional nilpotent Lie algebras (cf. Gon98]), for which the corresponding csc nilpotent Lie groups are not distinguished by Pansu's theorem [Pan89, Théorème 3], it holds for five of the families that all (but at most finitely many) members have pairwise isomorphic cohomology algebras, but that the cohomology algebras of the two remaining families fall in uncountably many 
isomorphism classes, thus providing new examples of uncountable families of connected simply connected nilpotent Lie groups which are pairwise non-quasi-isometric.

Standing assumptions. Unless otherwise specified, all generic vector spaces will be over the reals. So if $(X, \mu)$ is a measure space, then $L^{2}(X)$ will denote the Hilbert space of real valued square integrable functions, and similarly for other function spaces. We remark that this convention is primarily chosen to streamline notation, and that Theorem $\mathrm{A}$. Theorem $\mathrm{B}$ and Corollary [C hold verbatim over the complex numbers as well; see Remarks 3.2 and 4.5 .

Acknowledgements. The authors gratefully acknowledge the financial support from the Villum Foundation (grant no. 7423) and from the Independent Research Fund Denmark (grant no. 7014-00145B and 9040-00107B). They also thank Henrik D. Petersen whose earlier joint work with D.K. formed the basis for part of the present paper, Yves de Cornulier for suggesting the applications regarding quasi-isometry classification of 7-dimensional csc nilpotent Lie groups, and Nicky Cordua Mattsson for his assistance with programming issues in Maple. Finally, the authors would also like to thank the two anonymous referees for many helpful comments and suggestions; in particular we are grateful for the valuable input regarding the applications to nilpotent Lie groups, which has improved the quality of Section 5 significantly.

\section{Preliminaries}

2.1. Group cohomology. In this section we recall the basics on cohomology theory for locally compact groups, from the point of view of relative homological algebra; the reader is referred to [Gui80] for more details and proofs of the statements below. In what follows, $G$ denotes a locally compact second countable group.

Definition 2.1. A (continuous) $G$-module is a Hausdorff topological vector space $\mathcal{E}$ endowed with an action of $G$ by linear maps such that the action map $G \times \mathcal{E} \rightarrow \mathcal{E}$ is continuous. If $\mathcal{E}$ and $\mathcal{F}$ are $G$-modules, then a linear, continuous, $G$-equivariant map $\varphi: \mathcal{E} \rightarrow \mathcal{F}$ is called a morphism of $G$-modules and $\varphi$ is said to be strengthened if there exists a linear, continuous $\operatorname{map} \eta: \mathcal{F} \rightarrow \mathcal{E}$ such that $\varphi \circ \eta \circ \varphi=\varphi$.

Note that the map $\eta$ in the definition of a strengthened morphism is not required to be $G$-equivariant. The definition of a strengthened morphism given above is easily seen to be equivalent to the more standard formulation given, for instance, in [Gui80, Definition D.1].

Definition 2.2. A $G$-module $\mathcal{J}$ is said to be relatively injective if for any strengthened, oneto-one homomorphism $\iota: \mathcal{E} \rightarrow \mathcal{F}$ of $G$-modules $\mathcal{E}$ and $\mathcal{F}$ and any morphism $\varphi: \mathcal{E} \rightarrow \mathcal{J}$ there exists a morphism $\tilde{\varphi}: \mathcal{F} \rightarrow \mathcal{J}$ making the following diagram commutative

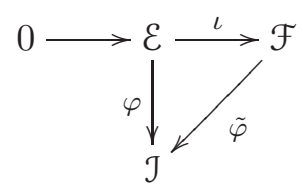

Definition 2.3. Let $\mathcal{E}$ be a $G$-module. A strengthened, relatively injective resolution of $\mathcal{E}$ is an exact complex

$$
0 \longrightarrow \mathcal{E} \stackrel{\iota}{\longrightarrow} \mathrm{J}^{0} \stackrel{d^{0}}{\longrightarrow} \mathrm{J}^{1} \stackrel{d^{1}}{\longrightarrow} \mathrm{J}^{2} \stackrel{d^{2}}{\longrightarrow} \cdots
$$

such that each $\mathcal{J}^{n}$ is a relatively injective $G$-module and $\iota$ and $d^{n}$ are strengthened morphisms. 
If $\left(\mathcal{J}^{n}, d^{n}\right)$ is a strengthened, relatively injective resolution of a $G$-module $\mathcal{E}$, then the cohomology, $\mathrm{H}^{n}(G, E)$, and reduced cohomology, $\underline{\mathrm{H}}^{n}(G, E)$, of $G$ with coefficients in $\mathcal{E}$ is defined as that of the complex

$$
0 \longrightarrow\left(\mathcal{J}^{0}\right)^{G} \stackrel{d^{0} \uparrow}{\longrightarrow}\left(\mathcal{J}^{1}\right)^{G} \stackrel{d^{1} \uparrow}{\longrightarrow}\left(\mathcal{J}^{2}\right)^{G} \stackrel{d^{2} \uparrow}{\longrightarrow} \cdots
$$

obtained by taking $G$-invariants; i.e.

$$
\mathrm{H}^{n}(G, \mathcal{E})=\frac{\operatorname{ker}\left(d^{n} \uparrow\right)}{\operatorname{im}\left(d^{n-1} \uparrow\right)} \text { and } \underline{H}^{n}(G, E)=\frac{\operatorname{ker}\left(d^{n} \uparrow\right)}{\operatorname{cl}\left(\operatorname{im}\left(d^{n-1} \uparrow\right)\right)},
$$

where $\mathrm{cl}(-)$ denotes closure in the topological vector space $\mathfrak{J}^{n}$. We will denote by $\mathrm{H}^{*}(G, \mathcal{E})$ and $\underline{\mathrm{H}}^{*}(G, \mathcal{E})$ the direct sums $\oplus_{n \geqslant 0} \mathrm{H}^{n}(G, \mathcal{E})$ and $\oplus_{n \geqslant 0} \underline{\mathrm{H}}^{n}(G, \mathcal{E})$, respectively. A standard argument Gui80, Corollaire 3.1.1] shows that $\mathrm{H}^{n}(G, \mathcal{E})$ is independent of the choice of strengthened, relative injective resolution up to isomorphism of (not necessarily Hausdorff!) topological vector spaces and hence also $\underline{\mathrm{H}}^{n}(G, E) \simeq \mathrm{H}^{n}(G, E) / \mathrm{cl}(\{0\})$ is independent of the choice of resolution. For the cohomology to be well defined one of course needs to know that strengthened, relatively injective resolutions exist, but in [Gui80, III, Proposition 1.2] it is shown that the complex

$$
0 \longrightarrow \mathcal{E} \stackrel{\iota}{\longrightarrow} C(G, \mathcal{E}) \stackrel{d^{0}}{\longrightarrow} C\left(G^{2}, \mathcal{E}\right) \stackrel{d^{1}}{\longrightarrow} C\left(G^{3}, \mathcal{E}\right) \stackrel{d^{2}}{\longrightarrow} \cdots
$$

with

$$
d^{n}(f)\left(g_{0}, \ldots, g_{n+1}\right)=\sum_{i=0}^{n+1}(-1)^{i} f\left(g_{0}, \ldots, \hat{g}_{i}, \ldots, g_{n+1}\right)
$$

constitutes such a resolution. Here the circumflex denotes omission and $C\left(G^{n}, \mathcal{E}\right)$ denotes the space of continuous functions from $G^{n}$ to $\mathcal{E}$ endowed with $G$-action given by

$$
(g . f)\left(g_{0}, \ldots, g_{n}\right):=g\left(f\left(g^{-1} g_{0}, \ldots, g^{-1} g_{n}\right)\right) .
$$

When $\mathcal{E}$ is a Fréchet space and $X$ is a $\sigma$-compact, locally compact, topological space whose Borel $\sigma$-algebra is endowed with a measure $\mu$, then one defines, for $p \in \mathbb{N}, \mathcal{L}_{\text {loc }}^{p}(X, \mathcal{E})$ as those measurable functions $f: X \rightarrow \mathcal{E}$ such that

$$
\int_{K} q(f(x))^{p} \mathrm{~d} \mu(x)<\infty
$$

for every compact set $K \subset X$ and every continuous seminorm $q$ on $\mathcal{E}$, and $L_{\mathrm{loc}}^{p}(X, \mathcal{E})$ is then defined by identifying functions in $\mathcal{L}_{\text {loc }}^{p}(X, \mathcal{E})$ that are equal $\mu$-almost everywhere. This too is a Fréchet space when endowed with the topology defined by the family of seminorms

$$
Q_{K, q}(f):=\sqrt[p]{\int_{K} q(f(x))^{p} \mathrm{~d} \mu(x)},
$$

where $K$ runs through the compact subsets of $X$ and $q$ runs through the continuous seminorms on $\mathcal{E}$. As lcsc groups are $\sigma$-compact this construction applies to $G$ and its powers and it was shown in Bla79, Corollaire 3.5] that the complex

$$
0 \longrightarrow \mathcal{E} \stackrel{\iota}{\longrightarrow} L_{\mathrm{loc}}^{p}(G, \mathcal{E}) \stackrel{d^{0}}{\longrightarrow} L_{\mathrm{loc}}^{p}\left(G^{2}, \mathcal{E}\right) \stackrel{d^{1}}{\longrightarrow} L_{\mathrm{loc}}^{p}\left(G^{3}, \mathcal{E}\right) \stackrel{d^{2}}{\longrightarrow} \cdots,
$$

with $G$-action and coboundary maps given by the natural analogues of (2) and (11), is also a strengthened, relatively injective resolution. 
2.1.1. Property $H_{T}$. Recall that a lcsc group $G$ is said to have Shalom's property $H_{T}$ (see [Sha04]) if for any unitary, complex Hilbert $G$-module $\mathcal{H}$ (i.e. $\mathcal{H}$ is a complex Hilbert space endowed with the structure of a continuous $G$-module and each element in $G$ acts as a unitary operator) the inclusion $\mathcal{H}^{G} \hookrightarrow \mathcal{H}$ induces an isomorphism $\underline{\mathrm{H}}^{1}\left(G, \mathcal{H}^{G}\right) \simeq \underline{\mathrm{H}}^{1}(G, \mathcal{H})$ or, equivalently, if $\underline{\mathrm{H}}^{1}(G, \mathcal{H})=\{0\}$ for every unitary $G$-module $\mathcal{H}$ with no non-trivial fixed-points. In this spirit, we will say that a group has property $H_{T}^{n}$ if $\underline{\mathrm{H}}^{n}(G, \mathcal{H})=\{0\}$ for every (complex) unitary $G$-module $\mathcal{H}$ with no non-trivial fixed-points. Since our main focus in the present paper is on groups acting on real Hilbert spaces, we remark at this point that a group has property $H_{T}^{n}$ if and only if it has the analogously defined property for real Hilbert spaces; this is easily seen by applying restriction of scalars and complexification, respectively.

Note that it follows by general disintegration theory and [Bla79, Theorem 10.1] that csc nilpotent Lie groups have property $H_{T}^{n}$ for all $n$, and from this and Mal'cev theory [Mal51 it also follows that torsion free, finitely generated nilpotent groups have property $H_{T}^{n}$ for all $n$; see [Sha04, Theorem 4.1.3] for more details.

2.1.2. Betti numbers. The Betti numbers of a group $G$ are defined as $\beta^{n}(G):=\operatorname{dim}_{\mathbb{R}} \mathrm{H}^{n}(G, \mathbb{R})$ and we remark that these are finite whenever $G$ is a csc nilpotent Lie group or a torsion free, discrete nilpotent group. For the latter, note that the classifying space of such groups are finite CW-complexes and the group cohomology agrees with the cohomology of the classifying space, and the former can be seen for instance by passing to Lie algebra cohomology via the van Est theorem vE55. Moreover, when $\beta^{n}(G)<\infty$, then $\mathrm{H}^{n}(G, \mathbb{R})$ is automatically Hausdorff [Gui80, III, Proposition 2.4] and hence there is no difference between reduced and ordinary cohomology.

2.1.3. Cup products. For a discrete group $\Gamma$, it is well-known that its cohomology with real coefficients $\mathrm{H}^{*}(\Gamma, \mathbb{R})=\oplus_{n \geqslant 0} \mathrm{H}^{n}(\Gamma, \mathbb{R})$ becomes a unital, graded-commutative $\mathbb{R}$-algebra for the so-called cup product, and the same construction also works for lcsc groups, but since this does not seem properly documented in the existing literature, we recall the construction below. If $G$ is lcsc groups and $\xi \in C\left(G^{n+1}, \mathbb{R}\right)$ and $\eta \in C\left(G^{m+1}, \mathbb{R}\right)$, one defines their cup product $\xi \smile \eta \in C\left(G^{n+m+1}, \mathbb{R}\right)$ as

$$
(\xi \smile \eta)\left(g_{0}, \ldots, g_{n+m}\right):=\xi\left(g_{0}, \ldots, g_{n}\right) \eta\left(g_{n}, \ldots, g_{n+m}\right) .
$$

As the cup product commutes with the (left regular) G-action, in the sense that $(g . \xi) \smile$ $(g . \eta)=g \cdot(\xi \smile \eta)$, it descends to a map

$$
\smile: C\left(G^{n+1}, \mathbb{R}\right)^{G} \times C\left(G^{m+1}, \mathbb{R}\right)^{G} \longrightarrow C\left(G^{n+m+1}, \mathbb{R}\right)^{G} .
$$

Moreover, the standard differentials (11) satisfy a graded Leibniz rule, i.e.

$$
d^{n+m}(\xi \smile \eta)=d^{n}(\xi) \smile \eta+(-1)^{n} \xi \smile d^{m}(\eta),
$$

from which it follows that the cup product passes down to the level of cocycles and that the set of coboundaries is an ideal, so that the cup product descends to a map

$$
\smile: \mathrm{H}^{n}(G, \mathbb{R}) \times \mathrm{H}^{m}(G, \mathbb{R}) \longrightarrow \mathrm{H}^{n+m}(G, \mathbb{R}),
$$

which turns $\mathrm{H}^{*}(G, \mathbb{R})$ into a unital, graded-commutative $\mathbb{R}$-algebra. We will encounter more elaborate cup products in Section 4 satisfying natural analogues of (4), so to make the argument easily available we now give the short proof of (4), in order to leave the details later to 
the reader in good conscience. To prove (4), let cocycles $\xi \in C\left(G^{n+1}, \mathbb{R}\right), \eta \in C\left(G^{m+1}, \mathbb{R}\right)$ be given and note that

$$
\begin{aligned}
& d^{n+m}(\xi \smile \eta)\left(g_{0} \ldots g_{n+m+1}\right)=\sum_{i=0}^{n+m+1}(-1)^{i}(\xi \smile \eta)\left(g_{0} \ldots \hat{g}_{i} \ldots g_{n+m+1}\right)= \\
& =\sum_{i=0}^{n}(-1)^{i} \xi\left(g_{0} \ldots \hat{g}_{i} \ldots g_{n+1}\right) \eta\left(g_{n+1} \ldots g_{n+m+1}\right)+\sum_{i=n+1}^{n+m+1}(-1)^{i} \xi\left(g_{0} \ldots g_{n}\right) \eta\left(g_{n} \ldots \hat{g}_{i} \ldots g_{n+m+1}\right) \\
& =\sum_{i=0}^{n}(-1)^{i} \xi\left(g_{0} \ldots \hat{g}_{i} \ldots g_{n+1}\right) \eta\left(g_{n+1} \ldots g_{n+m+1}\right)+\sum_{i=1}^{m+1}(-1)^{i+n} \xi\left(g_{0} \ldots g_{n}\right) \eta\left(g_{n} \ldots \hat{g}_{n+i} \ldots g_{n+m+1}\right)
\end{aligned}
$$

On the other hand

$$
\begin{aligned}
& \left(d^{n}(\xi) \smile \eta+(-1)^{n} \xi \smile d^{m}(\eta)\right)\left(g_{0} \ldots g_{n+m+1}\right)= \\
& =\sum_{i=0}^{n+1}(-1)^{i} \xi\left(g_{0} \ldots \hat{g}_{i} \ldots g_{n+1}\right) \eta\left(g_{n+1} \ldots g_{n+m+1}\right)+(-1)^{n} \sum_{i=0}^{m+1}(-1)^{i} \xi\left(g_{0} \ldots g_{n}\right) \eta\left(g_{n} \ldots \hat{g}_{n+i} \ldots g_{n+m+1}\right) \\
& =\sum_{i=0}^{n}(-1)^{i} \xi\left(g_{0} \ldots \hat{g}_{i} \ldots g_{n+1}\right) \eta\left(g_{n+1} \ldots g_{n+m+1}\right)+(-1)^{n} \sum_{i=1}^{m+1}(-1)^{i} \xi\left(g_{0} \ldots g_{n}\right) \eta\left(g_{n} \ldots \hat{g}_{n+i} \ldots g_{n+m+1}\right),
\end{aligned}
$$

where the last equality follows since the last summand in the first sum cancels with the first summand in the second; this proves (4).

A direct computation shows that the cup product is continuous with respect to the topology of uniform convergence on compacts at the level of cochains, and since we just saw that the coboundaries constitute an ideal, the same is true for the their closure. Thus, the cup product also descends to a product on $\underline{\mathrm{H}}^{*}(G, \mathbb{R})$.

2.2. Measure equivalence. In this section we review the necessary theory concerning (uniform) measure equivalence for locally compact groups; we refer to [BFS13] and [KKR17] for more details on the involved notions.

Definition 2.4 ([BFS13] $)$. Two unimodular lcsc groups $G$ and $H$ with Haar measures $\lambda_{G}$ and $\lambda_{H}$ are said to be measure equivalent if there exist a standard Borel measure $G \times H$-space $(\Omega, \eta)$ and two standard Borel measure spaces $(X, \mu)$ and $(Y, \nu)$ such that:

(i) both $\mu$ and $\nu$ are finite measures and $\eta$ is non-zero;

(ii) there exists an isomorphism of measure $G$-spaces $i:\left(G \times Y, \lambda_{G} \times \nu\right) \longrightarrow(\Omega, \eta)$, where $\Omega$ is considered a measure $G$-space for the restricted action and $G \times Y$ is considered a measure $G$-space for the action $g \cdot\left(g^{\prime}, y\right)=\left(g g^{\prime}, y\right)$;

(iii) there exists an isomorphism of measure $H$-spaces $j:\left(H \times X, \lambda_{H} \times \mu\right) \rightarrow(\Omega, \eta)$, where $\Omega$ is considered a measure $H$-space for the restricted action and $H \times X$ is considered a measure $H$-space for the action $h .\left(h^{\prime}, x\right)=\left(h h^{\prime}, x\right)$.

A standard Borel space $(\Omega, \eta)$ with these properties is called a measure equivalence coupling between $G$ and $H$, and whenever needed we will specify the additional data by writing $(\Omega, \eta, X, \mu, Y, \nu, i, j)$. Note also that since the Haar measure is unique up to scaling, the existence of a measure equivalence coupling is independent of the choice of Haar measures on the two groups in question. 
Any measure equivalence coupling gives rise to measure preserving actions $G \curvearrowright(X, \mu)$ and $H \curvearrowright(Y, \nu)$ as well as two 1-cocycles $\omega_{G}: H \times Y \rightarrow G$ and $\omega_{H}: G \times X \rightarrow H$. These are defined almost everywhere by the relations

$$
\begin{aligned}
& i\left(g \omega_{G}(h, y)^{-1}, h \cdot y\right)=h \cdot i(g, y), \quad \text { for almost all } g \in G . \\
& j\left(h \omega_{H}(g, x)^{-1}, g \cdot x\right)=g . j(h, x), \quad \text { for almost all } h \in H .
\end{aligned}
$$

In the definition of the actions and cocycles above, we are paying little attention to the measure theoretical subtleties, but the reader may find these worked out in detail in [KKR17, Section 2]. Note that it was was also proven in [KKR17] that one can always obtain a strict measure equivalence coupling; i.e. one in which the maps $i$ and $j$ are Borel isomorphisms and globally equivariant.

Definition 2.5 ([KKR17]). A strict measure equivalence coupling $(\Omega, \eta, X, \mu, Y, \nu, i, j)$ between unimodular, lcsc groups $G$ and $H$ is said to be uniform if

(i) for every compact $C \subset G$ there exists a compact $D \subset H$ such that $j^{-1} \circ i(C \times Y) \subset$ $D \times X$

(ii) for every compact $D \subset H$ there exists a compact $C \subset G$ such that $i^{-1} \circ j(D \times X) \subset$ $C \times Y$.

In this case $G$ and $H$ are said to be uniformly measure equivalent (UME), and the properties (i) and (ii) are referred to as the cocycles being locally bounded.

As mentioned already, measure equivalence was originally introduced by Gromov as a measure theoretic analogue of quasi-isometry, and although neither property in general implies the other, it was proven in [KKR17, Proposition 6.13] that for compactly generated], unimodular, lcsc groups, uniform measure equivalence always implies quasi-isometry, and that the converse holds under the additional assumption of amenability [KKR17, Theorem 6.15]. This generalises earlier results for discrete groups by Shalom [Sha04] and Sauer [Sau06].

If $(\Omega, \eta, X, \mu, Y, \nu, i, j)$ is a strict UME coupling between $G$ and $H$ and $\mathcal{E}$ is a Fréchet space then we define $L_{\mathrm{loc}}^{p}(\Omega, \mathcal{E})$ as those (equivalence classes modulo equality $\eta$-almost everywhere) of measurable functions $f: \Omega \rightarrow \mathcal{E}$ such that for every $C \subset G$ compact and every continuous seminorm $q$ on $\mathcal{E}$, one has

$$
\int_{C \times Y} q(f \circ i(g, y))^{2} \mathrm{~d} \lambda_{G}(g) \mathrm{d} \nu(y)<\infty,
$$

We topologise $L_{\mathrm{loc}}^{2}(\Omega, \mathcal{E})$ via the seminorms $q_{C}(f):=\sqrt{\int_{C \times Y} q(f \circ i(g, y))^{2} \mathrm{~d} \lambda_{G}(g) \mathrm{d} \nu(y)}$ and endow it with the $G \times H$ - action

$$
((g, h) . f)(t):=(g, h) . f\left((g, h)^{-1} . t\right), t \in \Omega,(g, h) \in G \times H .
$$

We could of course also have defined $L_{\text {loc }}^{p}(\Omega, \mathcal{E})$ using the map $j$ instead of $i$, but since $\Omega$ is uniform this gives rise to exactly the same space. To see this, take $f, C$ and $q$ as above. By local boundedness of the cocycles, we can find $D \subset H$ compact, such that $j^{-1} \circ i(C \times Y) \subset D \times X$ and consequently

$$
\int_{C \times Y} q(f \circ i(g, y))^{2} \mathrm{~d} \lambda_{G}(g) \mathrm{d} \nu(y)=\int_{C \times Y} q\left(f \circ j \circ j^{-1} \circ i(g, y)\right)^{2} \mathrm{~d} \lambda_{G}(g) \mathrm{d} \nu(y)
$$

\footnotetext{
${ }^{1}$ Recall that for compactly generated groups coarse equivalence coincides with quasi-isometry
} 


$$
\begin{aligned}
& =\int_{j^{-1} \circ i(C \times Y)} q(f \circ j(h, x))^{2} \mathrm{~d} \lambda_{H}(h) \mathrm{d} \mu(x) \\
& \leqslant \int_{D \times Y} q(f \circ j(h, x))^{2} \mathrm{~d} \lambda_{H}(h) \mathrm{d} \mu(x) .
\end{aligned}
$$

This shows that each of the seminorms defining the topology via the isomorphism $i$, is dominated by one of the seminorms defined via $j$, and by symmetry the converse is true as well, and the two families therefore generate the same topology on $L_{\mathrm{loc}}^{2}(\Omega, \mathcal{E})$.

Lemma 2.6. With the action defined by (5) the space $L_{\text {loc }}^{2}(\Omega, \mathcal{E})$ becomes a Fréchet $G \times H$ module.

Proof. Since $Y$ is standard Borel we may equip it with a compact metrizable topology generating the $\sigma$-algebra [Kec95, Theorem 15.6], and the space $L_{\text {loc }}^{2}(\Omega, \mathcal{E})$ then directly identifies with $L_{\text {loc }}^{2}(G \times Y, \mathcal{E})$ which is Fréchet by [Gui80, D.2]. It suffices to show that both groups act continuously, and by symmetry it is enough to treat the $G$-action. To this end, note that Gui80, D.2.2 (vii)] gives an isomorphism of $G$-modules

$$
L_{\mathrm{loc}}^{2}(\Omega, \mathcal{E}) \simeq L_{\mathrm{loc}}^{2}\left(G, L^{2}(Y, \mathcal{E})\right),
$$

where the action on the right hand side is given by $(g \cdot \xi)\left(g^{\prime}\right)(y)=g \cdot\left(\xi\left(g^{-1} g^{\prime}\right)(y)\right)$, so by Gui80, D.3.2] it suffices to show that the pointwise $G$-action on $L^{2}(Y, \mathcal{E})$ is continuous. To see this, note that $C(Y, \mathcal{E})$ is dense in $L^{2}(Y, \mathcal{E})$, so by [Gui80, Lemme D.8 (ii)], it is enough to show that the action is equicontinuous over compact sets and pointwise continuous on elements from $C(Y, \mathcal{E})$. To see the former, let $K \subset G$ be compact and $q$ be a continuous seminorm on $\mathcal{E}$. Then since $G \curvearrowright \mathcal{E}$ is continuous, there exists a continuous seminorm $q^{\prime}$ on $\mathcal{E}$ such that for all $g \in K$ and $x \in \mathcal{E}$ we have $q(g . x) \leqslant q^{\prime}(x)$. Hence, for $g \in K$ and $\xi \in L^{2}(Y, \mathcal{E})$ we also have that

$$
\int_{Y} q((g \cdot \xi)(y))^{2} \mathrm{~d} \nu(y)=\int_{Y} q(g \cdot \xi(y))^{2} \mathrm{~d} \nu(y) \leqslant \int_{Y} q^{\prime}(\xi(y))^{2} \mathrm{~d} \nu(y)
$$

showing equicontinuity over compact sets. To see that the action is pointwise continuous on $\xi \in C(Y, \mathcal{E})$, simply note that if $g_{n} \rightarrow g$ in $G$ and $q$ is a continuous seminorm on $\mathcal{E}$ then

$$
\begin{aligned}
\int_{Y}\left(q\left(g_{n} \cdot \xi-g \xi\right)(y)\right)^{2} \mathrm{~d} \nu(y) & =\int_{Y}\left(q\left(g_{n} \cdot \xi(y)-g \cdot \xi(y)\right)^{2} \mathrm{~d} \nu(y)\right. \\
& \leqslant \nu(Y) \sup _{x \in \xi(Y)} q\left(g_{n} \cdot x-g \cdot x\right)^{2} \underset{n \rightarrow \infty}{\longrightarrow} 0,
\end{aligned}
$$

where the convergence follows from compactness of $\xi(Y) \subset \mathcal{E}$ and [Gui80, Lemme D.8 (iii)].

\section{Proof of Theorem $\mathrm{A}$}

The aim of the current section is to prove Theorem $\mathrm{A}$, so we fix uniformly measure equivalent, unimodular lcsc groups $G$ and $H$ and a strict, uniform measure equivalence coupling $(\Omega, \eta, X \mu, Y, \nu, i, j)$ between them, as well as Fréchet $G \times H$-module $\mathcal{E}$. Consider the space $L_{\mathrm{loc}}^{2}\left(G^{n}, L_{\text {loc }}^{2}(\Omega, \mathcal{E})\right)$ endowed with $G \times H$-action

$$
((g, h) . \xi)\left(g_{1}, \ldots, g_{n}\right)(t):=(g, h)\left[\xi\left(g^{-1} g_{1}, \ldots, g^{-1} g_{n}\right)\left((g, h)^{-1} . t\right)\right],
$$

for $(g, h) \in G \times H, g_{1}, \ldots, g_{n} \in G, t \in \Omega$. 
Lemma 3.1. The space $L_{\mathrm{loc}}^{2}\left(G^{n}, L_{\mathrm{loc}}^{2}(\Omega, \mathcal{E})\right)$ is a relatively injective $G \times H$-module and the complex $R_{G}$ defined as

$$
0 \longrightarrow L_{\mathrm{loc}}^{2}(\Omega, \mathcal{E}) \longrightarrow L_{\mathrm{loc}}^{2}\left(G, L_{\mathrm{loc}}^{2}(\Omega, \mathcal{E})\right) \longrightarrow L_{\mathrm{loc}}^{2}\left(G^{2}, L_{\mathrm{loc}}^{2}(\Omega, \mathcal{E})\right) \longrightarrow \cdots
$$

constitutes a strengthened, relatively injective resolution of the $G \times H$-module $L_{\text {loc }}^{2}(\Omega, \mathcal{E})$ when endowed with the standard homogeneous differentials, given by the obvious extension of the formula (11).

Proof. Throughout the proof we identify $\Omega$ with $H \times X$ through the map $j$ defining the $L_{\text {loc }}^{2}$-structure on $\Omega$; recall that under this identification the $G \times H$-action on

$$
L_{\mathrm{loc}}^{2}\left(G^{n+1}, L_{\mathrm{loc}}^{2}(\Omega, \mathcal{E})\right)=L_{\mathrm{loc}}^{2}\left(G^{n+1}, L_{\mathrm{loc}}^{2}(H \times X, \mathcal{E})\right)
$$

is given by the formula

$$
((g, h) \cdot \xi)\left(g_{0}, \ldots, g_{n}\right)\left(h^{\prime}, x\right)=(g, h) .\left[\xi\left(g^{-1} g_{0}, \ldots, g^{-1} g_{n}\right)\left(h^{-1} h^{\prime} \omega_{H}\left(g^{-1}, x\right)^{-1}, g^{-1} \cdot x\right)\right] .
$$

Since $X$ is standard Borel, [Kec95, Theorem 15.6] ensures that we can find a compact, metrizable topology on it whose open sets generate the Borel structure, and we may therefore consider the Fréchet space $L_{\text {loc }}^{2}\left(G^{n} \times X, \mathcal{E}\right)$. Arguing as in [Gui80, $\mathrm{n}^{\circ}$ D.3.2.], we obtain that this is a Fréchet $G \times H$-module when endowed with the $G \times H$-action

$$
((g, h) \cdot \xi)\left(g_{1}, \ldots, g_{n}, x\right):=(g, h) \cdot \xi\left(g^{-1} g_{1}, \ldots, g^{-1} g_{n}, g^{-1} \cdot x\right),
$$

and by [Bla79, Théorème 3.4], we therefore have that

$$
L_{\mathrm{loc}}^{2}\left(G \times H, L_{\mathrm{loc}}^{2}\left(G^{n} \times X, \mathcal{E}\right)\right),
$$

with the standard $G \times H$-action, is a relatively injective Fréchet $G \times H$-module. A routine calculation now shows that the map

$$
\begin{aligned}
\alpha: L_{\mathrm{loc}}^{2}\left(G^{n+1}, L_{\mathrm{loc}}^{2}(H \times X, \mathcal{E})\right) & \longrightarrow L_{\mathrm{loc}}^{2}\left(G \times H, L_{\mathrm{loc}}^{2}\left(G^{n} \times X, \mathcal{E}\right)\right) \\
\alpha(\xi)(g, h)\left(g_{1}, \ldots, g_{n}, x\right) & :=\xi\left(g_{1}, \ldots, g_{n}, g\right)\left(h \omega_{H}\left(g^{-1}, x\right), x\right),
\end{aligned}
$$

is an isomorphism of Fréchet spaces intertwining the $G \times H$-actions, and hence it follows that also $L_{\text {loc }}^{2}\left(G^{n+1}, L_{\text {loc }}^{2}(\Omega, \mathcal{E})\right)$ is relatively injective; we will here only show equivariance of $\alpha$, leaving the verification of invertibility and continuity to the reader: Acting with $(\bar{g}, \bar{h}) \in G \times H$ before and after applying $\alpha$ gives

$$
\begin{aligned}
& \alpha((\bar{g}, \bar{h}) \cdot \xi)(g, h)\left(g_{1}, \ldots, g_{n-1}, x\right)=((\bar{g}, \bar{h}) \cdot \xi)\left(g_{1}, \ldots, g_{n}, g\right)\left(h \omega_{H}\left(g^{-1}, x\right), x\right) \\
= & (\bar{g}, \bar{h}) .\left[\xi\left(\bar{g}^{-1} g_{1}, \ldots, \bar{g}^{-1} g_{n-1}, \bar{g}^{-1} g\right)\left(\bar{h}^{-1} h \omega_{H}\left(g^{-1}, x\right) \omega_{H}\left(\bar{g}^{-1}, x\right)^{-1}, \bar{g}^{-1} x\right)\right],
\end{aligned}
$$

and

$$
\begin{array}{r}
((\bar{g}, \bar{h}) . \alpha(\xi))(g, h)\left(g_{1}, \ldots, g_{n-1}, x\right)=(\bar{g}, \bar{h}) \cdot\left[\alpha(\xi)\left(\bar{g}^{-1} g, \bar{h}^{-1} h\right)\left(\bar{g}^{-1} g_{1}, \ldots, \bar{g}^{-1} g_{n-1}, \bar{g}^{-1} x\right)\right] \\
=(\bar{g}, \bar{h})\left[\xi\left(\bar{g}^{-1} g_{1}, \ldots, \bar{g}^{-1} g_{n-1}, \bar{g}^{-1} g\right)\left(\bar{h}^{-1} h \omega_{H}\left(g^{-1} \bar{g}, \bar{g}^{-1} x\right), \bar{g}^{-1} x\right)\right],
\end{array}
$$

respectively. The result now follows, since the cocycle identity implies

$$
\omega_{H}\left(g^{-1} \bar{g}, \bar{g}^{-1} x\right) \omega_{H}\left(\bar{g}^{-1}, x\right)=\omega_{H}\left(g^{-1}, x\right) .
$$

Since the complex $R_{G}$ is simply the standard $L_{\text {loc }}^{2}$-resolution of $L_{\text {loc }}^{2}(\Omega, \mathcal{E})$ considered only as a $G$-module, it is clearly strengthened, and hence the proof is complete. 
Proof of Theorem $A$. Since the roles of $G$ and $H$ are symmetric in the module structure on $L_{\text {loc }}^{2}(\Omega, \mathcal{E})$ one may construct a strengthened, relatively injective resolution $R_{H}$ of $L_{\text {loc }}^{2}(\Omega, \mathcal{E})$ analogous to (6), whose degree $n$ term is given by $L_{\text {loc }}^{2}\left(H^{n+1}, L_{\text {loc }}^{2}(\Omega, \varepsilon)\right)$. Thus, both $R_{G}$ and $R_{H}$ compute $\mathrm{H}^{n}\left(G \times H, L_{\text {loc }}^{2}(\Omega, \mathcal{E})\right)$ after passing to $G \times H$-invariants and cohomology. However,

$$
L_{\mathrm{loc}}^{2}\left(G^{n+1}, L_{\mathrm{loc}}^{2}(\Omega, \mathcal{E})\right)^{G \times H}=\left(L_{\mathrm{loc}}^{2}\left(G^{n+1}, L_{\mathrm{loc}}^{2}(\Omega, \mathcal{E})\right)^{H}\right)^{G}=\left(L_{\mathrm{loc}}^{2}\left(G^{n+1}, L_{\mathrm{loc}}^{2}(\Omega, \mathcal{E})^{H}\right)\right)^{G},
$$

where the last equality is due to the fact that $H$ acts trivially in the $G^{n+1}$-direction. From this we see that passing to $G \times H$-invariants in $R_{G}$ is exactly the same as passing to $G$-invariants in the $L_{\text {loc }}^{2}$-resolution (3) of the $G$-module $L_{\text {loc }}^{2}(\Omega, \mathcal{E})^{H}$, and hence we obtain a topological isomorphism

$$
\mathrm{H}^{n}\left(G, L_{\mathrm{loc}}^{2}(\Omega, \mathcal{E})^{H}\right) \simeq \mathrm{H}^{n}\left(G \times H, L_{\mathrm{loc}}^{2}(\Omega, \mathcal{E})\right) .
$$

Replacing $R_{G}$ with $R_{H}$, a symmetric argument yields that

$$
\mathrm{H}^{n}\left(H, L_{\mathrm{loc}}^{2}(\Omega, \mathcal{E})^{G}\right) \simeq \mathrm{H}^{n}\left(G \times H, L_{\mathrm{loc}}^{2}(\Omega, \mathcal{E})\right),
$$

and the desired isomorphism follows.

If $\mathcal{E}=\mathbb{R}$ with trivial $G \times H$-action, then by Gui80, D.2.2 (vii) \& Lemme D.9] we have an isomorphism of $H$-modules

$$
L_{\mathrm{loc}}^{2}(\Omega, \mathbb{R})^{G} \simeq L_{\mathrm{loc}}^{2}(G \times Y, \mathbb{R})^{G} \simeq L_{\mathrm{loc}}^{2}\left(G, L^{2}(Y)\right)^{G} \simeq L^{2}(Y)
$$

and, similarly, an isomorphism of $G$-modules $L_{\text {loc }}^{2}(\Omega, \mathcal{E})^{H} \simeq L^{2}(X)$; hence the last part of the statement follows from the first.

Remark 3.2. Theorem $\mathrm{A}$ is stated for real Fréchet spaces and real cohomology, but as seen from the proof just given, the analogous statement over the complex numbers also holds true with verbatim the same proof.

Remark 3.3. The proof just given does not, directly, provide a concrete map realizing the isomorphism $\mathrm{H}^{n}\left(G, L_{\text {loc }}^{2}(\Omega, \mathcal{E})^{H}\right) \simeq \mathrm{H}^{n}\left(H, L_{\mathrm{loc}}^{2}(\Omega, \mathcal{E})^{G}\right)$, but for applications, e.g. our Theorem $\mathrm{B}$, having a concrete map is very useful, and we shall therefore now describe one such a map. By general relative homological algebra [Gui80, III, Corollaire 1.1], any morphism $\chi$ of complexes of $G \times H$-modules from $R_{G}$ to $R_{H}$ which lifts the identity on $L_{\text {loc }}^{2}(\Omega, \mathcal{E})$ will induce a (topological) isomorphism $\mathrm{H}^{*}\left(R_{G}^{G \times H}, d^{n} \uparrow\right) \simeq \mathrm{H}^{*}\left(R_{H}^{G \times H}, d^{n} \uparrow\right)$. We now define such a map

$$
\chi^{n}: L_{\mathrm{loc}}^{2}\left(G^{n+1}, L_{\mathrm{loc}}^{2}(\Omega, \mathcal{E})\right) \longrightarrow L_{\mathrm{loc}}^{2}\left(H^{n+1}, L_{\mathrm{loc}}^{2}(\Omega, \mathcal{E})\right)
$$

by setting

$$
\chi^{n}(\xi)\left(h_{0}, \ldots, h_{n}\right)(t):=\xi\left(\pi_{G} \circ i^{-1}\left(h_{0}^{-1} . t\right), \ldots, \pi_{G} \circ i^{-1}\left(h_{n}^{-1} . t\right)\right)(t),
$$

where $\pi_{G}: G \times Y \rightarrow G$ denotes the projection onto the first factor. Identifying $\Omega$ with $G \times Y$ via $i$ the map takes the form

$$
\chi^{n}(\xi)\left(h_{0}, \ldots, h_{n}\right)(g, y)=\xi\left(g \omega_{G}\left(h_{0}^{-1}, y\right)^{-1}, \ldots, g \omega_{G}\left(h_{n}^{-1}, y\right)^{-1}\right)(g, y) .
$$

Note that the map $\chi^{n}$ does indeed take values in $L_{\text {loc }}^{2}\left(H^{n+1}, L_{\mathrm{loc}}^{2}(\Omega, \mathcal{E})\right)$ since $\Omega$ is uniform such that the cocycles are locally bounded, and from this it also follows that $\chi^{n}$ is continuous. It is straightforward to see that $\chi$ is a chain map lifting the identity on $L_{\text {loc }}^{2}(\Omega, \mathcal{E})$ and from the cocycle identity it follows that $\chi^{n}$ is $G \times H$-equivariant. 


\section{Proof of Theorem B}

In this section we prove Theorem $\mathrm{B}$. We thus assume that $G$ and $H$ are uniformly measure equivalent, unimodular, lcsc groups satisfying property $H_{T}^{n}$ for all $n$, and fix a strict, uniform measure equivalence coupling $(\Omega, \eta, X, \mu, Y, \nu, i, j)$. By [KKR17, Proposition 2.13], and its proof, one may change the measures $\eta, \mu$ and $\nu$ into ones that are ergodic for the $G \times H$-, $G$ - and $H$-actions, respectively, so we may, and shall, assume that the original measures are ergodic. Furthermore, by rescaling the measures involved, we may also assume that $\nu$ is a probability measure. We therefore obtain isomorphisms

$$
\begin{aligned}
\underline{\mathrm{H}}^{n}(G, \mathbb{R}) & =\underline{\mathrm{H}}^{n}\left(G, L^{2}(X)^{G}\right) \\
& \simeq \underline{\mathrm{H}}^{n}\left(G, L^{2}(X)\right) \\
& \simeq \underline{\mathrm{H}}^{n}\left(G, L_{\mathrm{loc}}^{2}(\Omega)^{H}\right) \\
& \simeq \underline{\mathrm{H}}^{n}\left(H, L_{\mathrm{loc}}^{2}(\Omega)^{G}\right) \\
& \simeq \underline{\mathrm{H}}^{n}\left(H, L^{2}(Y)\right) \\
& \simeq \underline{\mathrm{H}}^{n}(H, \mathbb{R})
\end{aligned}
$$

(Theorem @)

(ergodicity and property $H_{T}^{n}$ )

Chasing through the isomorphisms above, using the explicit isomorphism $\chi^{n}$ from Remark 3.3, shows that

$$
\kappa^{n}: L_{\mathrm{loc}}^{2}\left(G^{n+1}, \mathbb{R}\right) \longrightarrow L_{\mathrm{loc}}^{2}\left(H^{n+1}, \mathbb{R}\right),
$$

given by $\kappa^{n}(\xi)\left(h_{0}, \ldots, h_{n}\right)=\int_{Y} \xi\left(\omega_{G}\left(h_{0}^{-1}, y\right)^{-1}, \ldots, \omega_{G}\left(h_{n}^{-1}, y\right)^{-1}\right) \mathrm{d} \nu(y)$ at the cochain level induces the isomorphism $\underline{\mathrm{H}}^{n}(G, \mathbb{R}) \simeq \underline{\mathrm{H}}^{n}(H, \mathbb{R})$ and the aim is now to prove that this isomorphism preserves cup products. To this end, we need an auxiliary complex defined as follows:

Definition 4.1. Denote by $D^{n}$ the subspace of $L_{\text {loc }}^{2}\left(H^{n+1}, L^{2}(Y)\right)$ consisting of the classes (modulo equality almost everywhere) of functions $\xi \in \mathcal{L}_{\text {loc }}^{2}\left(H^{n+1}, L^{2}(Y)\right)$ such that:

(i) for almost all $\left(h_{0}, \ldots, h_{n}\right) \in H^{n+1}: \xi\left(h_{0}, \ldots, h_{n}\right) \in L^{\infty}(Y) \subset L^{2}(Y)$, and

(ii) for all $C \subset H^{n+1}$ compact: $\operatorname{ess} \sup \left\{\left\|\xi\left(h_{0}, \ldots, h_{n}\right)\right\|_{\infty}:\left(h_{0}, \ldots, h_{n}\right) \in C\right\}<\infty$.

Since $L^{\infty}(Y) \subset L^{2}(Y)$ is an $H$-invariant subspace, $D^{n}$ becomes a (non-complete) $H$ submodule of $L_{\text {loc }}^{2}\left(H^{n+1}, L^{2}(Y)\right)$. Moreover, it is easily seen that $d^{n}\left(D^{n}\right) \subseteq D^{n+1}$ and hence

$$
D:\left(D^{0}\right)^{H} \stackrel{d^{0} \uparrow}{\longrightarrow}\left(D^{1}\right)^{H} \stackrel{d^{1} \uparrow}{\longrightarrow}\left(D^{2}\right)^{H} \stackrel{d^{2} \uparrow}{\longrightarrow} \cdots
$$

is a subcomplex of the standard $L_{\text {loc }}^{2}$-complex computing $\mathrm{H}^{*}\left(H, L^{2}(Y)\right)$.

Definition 4.2. For $\alpha \in D^{n}, \beta \in D^{m}$ and $\xi \in L_{\text {loc }}^{2}\left(H^{n+1}, L^{2}(Y)\right)$ we define $\alpha \smile \xi, \xi \smile \alpha \in$ $L_{\text {loc }}^{2}\left(H^{m+n+1}, L^{2}(Y)\right)$ and $\alpha \smile \beta \in D^{n+m}$ by

$$
\begin{aligned}
& \alpha \smile \xi\left(h_{0}, \ldots, h_{n+m}\right):=\alpha\left(h_{0}, \ldots, h_{n}\right) \xi\left(h_{n}, \ldots, h_{n+m}\right) \\
& \xi \smile \alpha\left(h_{0}, \ldots, h_{n+m}\right):=\xi\left(h_{0}, \ldots, h_{m}\right) \alpha\left(h_{m}, \ldots, h_{n+m}\right) \\
& \alpha \smile \beta\left(h_{0}, \ldots, h_{n+m}\right):=\alpha\left(h_{0}, \ldots, h_{n}\right) \beta\left(h_{n}, \ldots, h_{n+m}\right)
\end{aligned}
$$

where the products on the right hand side are the pointwise products between functions in $L^{\infty}(Y)$ and $L^{2}(Y)$. 
Lemma 4.3. The product defined by (9) descends to a product on $\mathrm{H}^{*}(D)$ turning it into a unital $\mathbb{R}$-algebra. Similarly, the products (77) and (8) descend to the level of cohomology and reduced cohomology turning $\mathrm{H}^{*}\left(H, L^{2}(Y)\right)$ and $\underline{\mathrm{H}}^{*}\left(H, L^{2}(Y)\right)$ into bimodules for $\mathrm{H}^{*}(D)$.

Proof. For $f_{1} \in L^{\infty}(Y), f_{2} \in L^{2}(Y)$ and $h \in H$ one has

$$
h \cdot\left(f_{1} \cdot f_{2}\right)=\left(h \cdot f_{1}\right) \cdot\left(h \cdot f_{2}\right) \text { and } h \cdot\left(f_{2} \cdot f_{1}\right)=\left(h \cdot f_{2}\right) \cdot\left(h \cdot f_{1}\right)
$$

(the products being defined pointwise) and from this it follows that the cup product of $\mathrm{H}$ invariant elements is again $H$-invariant, so that $\smile$ restricts to the level of fixed points for $H$. Moreover, by repeating the proof of (44) mutatis mutandis, the cup products (7), (8) and (9), are seen to satisfy the obvious versions of the Leibniz rule, from which it follows that $\mathrm{H}^{*}(D)$ becomes a (graded) $\mathbb{R}$-algebra for which $\mathrm{H}^{*}\left(H, L^{2}(Y)\right)$ is a (graded) bimodule. We will omit the details of the latter, and instead show that $\underline{\mathrm{H}}^{*}\left(H, L^{2}(Y)\right)$ becomes a $\mathrm{H}^{*}(D)$ bimodule, since this proof contains the other as a special case. We need to show that if $\alpha, \alpha^{\prime} \in$ $D^{n} \cap \operatorname{ker}\left(d^{n}\right)$ and $\xi, \xi^{\prime} \in L_{\text {loc }}^{2}\left(H^{m+1}, L^{2}(Y)\right) \cap \operatorname{ker}\left(d^{m}\right)$ satisfy that $\alpha-\alpha^{\prime} \in d^{n-1}\left(D^{n-1}\right)$ and $\xi-\xi^{\prime} \in \operatorname{cl}\left(d^{m-1}\left(L_{\text {loc }}^{2}\left(H^{m}, L^{2}(Y)\right)\right)\right.$ then $\alpha \smile \xi-\alpha^{\prime} \smile \xi^{\prime} \in \operatorname{cl}\left(d^{n+m-1}\left(L_{\text {loc }}^{2}\left(H^{n+m}, L^{2}(Y)\right)\right)\right.$. Write $\alpha-\alpha^{\prime}=d^{n-1}(\beta)$ and note that, by the Leibniz rule, we have

$$
\begin{aligned}
\alpha \smile \xi-\alpha^{\prime} \smile \xi^{\prime} & =\left(\alpha-\alpha^{\prime}\right) \smile \xi-\alpha^{\prime} \smile\left(\xi-\xi^{\prime}\right) \\
& =d^{n-1}(\beta) \smile \xi-\alpha^{\prime} \smile\left(\xi-\xi^{\prime}\right) \\
& =d^{n+m-1}(\beta \smile \xi)-(-1)^{n-1} \beta \smile d^{m}(\xi)-\alpha^{\prime} \smile\left(\xi-\xi^{\prime}\right) \\
& =d^{n+m-1}(\beta \smile \xi)-\alpha^{\prime} \smile\left(\xi-\xi^{\prime}\right) .
\end{aligned}
$$

By assumption, there exist $\eta_{k} \in L_{\mathrm{loc}}^{2}\left(H^{m}, L^{2}(Y)\right)$ such that $\lim _{k} d^{m-1}\left(\eta_{k}\right)=\xi-\xi^{\prime}$, so to finish the proof it suffices to show that the cup product is pointwise continuous in the second variable; i.e. that if $\zeta_{k} \rightarrow_{k} 0$ in $L_{\mathrm{loc}}^{2}\left(H^{m+1}, L^{2}(Y)\right)$ then $\alpha \smile \zeta_{k} \rightarrow_{k} 0$ in $L_{\mathrm{loc}}^{2}\left(H^{m+n+1}, L^{2}(Y)\right)$. To see this, let a compact $K \subset H^{n+m+1}$ be given. Upon passing to a bigger compact set, we may assume that $K=\prod_{i=0}^{n+m} K_{i}$ for some compact subsets $K_{i} \subset H$ and we now get

$$
\begin{aligned}
& \int_{K}\left\|\alpha \smile \zeta_{k}\right\|_{2} \mathrm{~d} \lambda_{H}^{\otimes(n+m+1)}=\int_{\prod_{i=0}^{n+m} K_{i}}\left\|\alpha\left(h_{0}, \ldots, h_{n}\right) \zeta_{k}\left(h_{n}, \ldots, h_{n+m}\right)\right\| \mathrm{d} h_{0} \cdots \mathrm{d} h_{n+m} \\
& \leqslant \sup _{\left(h_{0}, \ldots, h_{n}\right) \in \prod_{i=0}^{n-1} K_{i}}\left\|\alpha\left(h_{0}, \ldots, h_{n}\right)\right\|_{\infty} \prod_{i=0}^{n} \lambda_{H}\left(K_{i}\right) \int_{\prod_{i=n}^{n+m} K_{i}}\left\|\zeta_{k}\left(h_{n}, \ldots, h_{n+m}\right)\right\| \mathrm{d} h_{n} \cdots \mathrm{d} h_{n+m} .
\end{aligned}
$$

By definition of $D^{n}$ we have

$$
\sup _{\left(h_{0}, \ldots, h_{n}\right) \in \prod_{i=0}^{n} K_{i}}\left\|\alpha\left(h_{0}, \ldots, h_{n}\right)\right\|_{\infty}<\infty,
$$

and by assumption

$$
\int_{\prod_{i=n}^{n+m} K_{i}}\left\|\zeta_{k}\left(h_{n}, \ldots, h_{n+m}\right)\right\| \mathrm{d} h_{n} \cdots \mathrm{d} h_{n+m} \rightarrow_{k} 0 .
$$

Remark 4.4. Using the usual contracting homotopy (cf. Gui80, III, Proposition 1.4]) for the resolution $\left(L_{\text {loc }}^{2}\left(H^{n+1}, L^{2}(Y)\right), d^{n}\right)_{n \in \mathbb{N}_{0}}$, one easily checks that $\left(D^{n}, d^{n}\right)_{n \in \mathbb{N}_{0}}$ is a strengthened resolution of the $H$-submodule $L^{\infty}(Y) \subset L^{2}(Y)$, but it seems less clear whether the modules $D^{n}$ are relatively injective or not. If this were the case, then $\mathrm{H}^{*}(D)$ would of course be nothing but $\mathrm{H}^{*}\left(H, L^{\infty}(Y)\right)$, where $L^{\infty}(Y)$ is considered as an $H$-module with respect to the 2-norm. 
With the lemmas above at our disposal, we can now prove Theorem $B$ following the strategy in [Sau06] almost verbatim. Recall that we have fixed a strict, ergodic UME coupling $(\Omega, \eta, X, \mu, Y, \nu, i, j)$ and normalised the measures so that $\nu$ has total mass 1 .

Proof of Theorem $B$. Denote by $L_{0}^{2}(Y)$ the functions in $L^{2}(Y)$ that integrate to zero. The Hilbert $H$-module $L^{2}(Y)$ then splits as an (orthogonal) direct sum $L^{2}(Y)=L_{0}^{2}(Y) \oplus \mathbb{R}$, via the map $\xi \mapsto\left(\xi-\left(\int_{Y} \xi \mathrm{d} \nu\right) 1_{Y}, \int_{Y} \xi \mathrm{d} \nu\right)$. From this it follows that we get a decomposition of $H$-complexes

$$
\Phi: L_{\mathrm{loc}}^{2}\left(H^{n+1}, L^{2}(Y)\right) \simeq L_{\mathrm{loc}}^{2}\left(H^{n+1}, L_{0}^{2}(Y)\right) \oplus L_{\mathrm{loc}}^{2}\left(H^{n+1}, \mathbb{R}\right),
$$

by simply composing a function $L_{\text {loc }}^{2}\left(H^{n+1}, L^{2}(Y)\right)$ with the two projections $p_{1}: L^{2}(Y) \rightarrow$ $L_{0}^{2}(Y)$ and $p_{2}: L^{2}(Y) \rightarrow \mathbb{R}$. Similarly, denote by $D_{0}^{n}$ the subcomplex of $D^{n}$ consisting of the (classes of) those functions that integrate to zero almost everywhere and by $D_{\mathbb{R}}^{n}$ the (classes of functions) that, almost everywhere, are almost everywhere constant on $Y$, and note that the decomposition $\Phi$ maps $D^{n}$ onto $D_{0}^{n} \oplus D_{\mathbb{R}}^{n}$. To be extremely precise, $D_{0}^{n}$ consists of classes of those $\xi \in \mathcal{L}_{\text {loc }}^{2}\left(H^{n+1}, L^{2}(Y)\right)$ such that $[\xi] \in D^{n}$ and such that for almost all $h_{0}, \ldots, h_{n}$ we have $\int_{Y} \xi\left(h_{0}, \ldots, h_{n}\right) d \nu=0$ and $D_{\mathbb{R}}^{n}$ are the classes of those functions $\xi \in \mathcal{L}_{\mathrm{loc}}^{2}\left(H^{n+1}, L^{2}(Y)\right)$ such that $[\xi] \in D^{n}$ and such that for almost all $h_{0}, \ldots, h_{n}$ we have $\xi\left(h_{0}, \ldots, h_{n}\right)=\left(\int_{Y} \xi\left(h_{0}, \ldots, h_{n}\right) d \nu\right) 1_{Y}$ (the latter equation in $L^{\infty}(Y) \subset L^{2}(Y)$ We therefore obtain splittings at the level of cohomology

$$
\begin{aligned}
\mathrm{H}^{n}(D) & \simeq \mathrm{H}^{n}\left(D_{0}\right) \oplus \mathrm{H}^{n}\left(D_{\mathbb{R}}\right) \\
\mathrm{H}^{n}\left(H, L^{2}(Y)\right) & \simeq \mathrm{H}^{n}\left(H, L_{0}^{2}(Y)\right) \oplus \mathrm{H}^{n}(H, \mathbb{R}) \\
\underline{\mathrm{H}}^{n}\left(H, L^{2}(Y)\right) & \simeq \underline{\mathrm{H}}^{n}\left(H, L_{0}^{2}(Y)\right) \oplus \underline{\mathrm{H}}^{n}(H, \mathbb{R})
\end{aligned}
$$

respecting the natural downward maps induced by the corresponding inclusions at the level of complexes. As shown in the first paragraph of the present section, we have a continuous linear map

$$
I^{*}: \mathrm{H}^{*}(G, \mathbb{R}) \longrightarrow \mathrm{H}^{*}\left(H, L^{2}(Y)\right),
$$

which descends to an isomorphism after the passage to reduced cohomology, and maps the (class of a) continuous $n$-cocycle $\xi \in C\left(G^{n+1}, \mathbb{R}\right)^{G} \cap \operatorname{ker}\left(d^{n}\right)$ to the (class of) the cocycle $I(\xi) \in L_{\text {loc }}^{2}\left(H^{n+1}, L^{2}(Y)\right)$ given by

$$
I(\xi)\left(h_{0}, \ldots, h_{n}\right)(y)=\xi\left(\omega_{G}\left(h_{0}^{-1}, y\right)^{-1}, \ldots, \omega_{G}\left(h_{n}^{-1}, y\right)^{-1}\right) .
$$

Moreover, since $\Omega$ is uniform, the cocycle $\omega_{G}$ is locally bounded and from this it follows that $I(\xi) \in D^{n}$, where $D^{n}$ is the submodule of $L_{\text {loc }}^{2}\left(H^{n+1}, L^{2}(Y)\right)$ described in Definition 4.1. Hence, $I^{*}$ factorises as

$$
\mathrm{H}^{*}(G, \mathbb{R}) \stackrel{I_{0}^{*}}{\longrightarrow} \mathrm{H}^{*}(D) \stackrel{\iota^{*}}{\longrightarrow} \mathrm{H}^{*}\left(H, L^{2}(Y)\right),
$$

where $\iota$ is the map induced by the inclusion $D^{n} \subset L_{\text {loc }}^{2}\left(H^{n+1}, L^{2}(Y)\right)$. Denoting by $\underline{\iota}^{*}$ the map obtained by composing $\iota^{*}: \mathrm{H}^{*}(D) \longrightarrow \mathrm{H}^{*}\left(H, L^{2}(Y)\right)$ with the natural projection $\mathrm{H}^{*}\left(H, L^{2}(Y)\right) \rightarrow \underline{\mathrm{H}}^{*}\left(H, L^{2}(Y)\right)$, we obtain a sequence of maps as follows:

$$
\mathrm{H}^{*}(G, \mathbb{R}) \stackrel{I_{0}^{*}}{\longrightarrow} \mathrm{H}^{*}(D) \stackrel{p^{*} \stackrel{\underline{\underline{L}}^{*}}{\longrightarrow} \underline{\mathrm{H}}^{*}\left(H, L^{2}(Y)\right) \stackrel{p_{2}^{*}}{\sim} \underline{\mathrm{H}}^{*}}{\longrightarrow}(H, \mathbb{R})
$$


Here $p_{2}^{*}$ is the map induced at the level of reduced cohomology by the map $L^{2}(Y) \rightarrow \mathbb{R}$ given by integration against $\nu$ and $p^{*}$ is simply defined as $p_{2}^{*} \circ \underline{\iota}^{*}$. Now, $I_{0}^{*}$ is multiplicative, since already at the level of cochains we have $I_{0}(\xi \smile \eta)=I_{0}(\xi) \smile I_{0}(\eta)$ for $\xi \in C\left(G^{n+1}, \mathbb{R}\right)$ and $\eta \in C\left(G^{m+1}, \mathbb{R}\right)$, which is seen by a direct computation.

We now aim to show that also $p^{*}=p_{2}^{*} \circ \underline{\iota}^{*}$ is multiplicative. To this end, first notice the map $\underline{\iota}^{*}$ is a bimodule map with respect to the $\mathrm{H}^{*}(D)$-bimodule structure on $\mathrm{H}^{*}(D)$ given by left/right multiplication and the $\mathrm{H}^{*}(D)$-bimodule structure on $\underline{\mathrm{H}}^{n}\left(H, L^{2}(Y)\right)$ described in Lemma 4.3, and its kernel is therefore a two-sided ideal. Moreover, since $p_{2}^{*}$ is an isomorphism, we have $\operatorname{ker}\left(\underline{\iota}^{*}\right)=\operatorname{ker}\left(p^{*}\right)$, and from this we now obtain that $p^{*}$ is multiplicative as follows: given $[\xi] \in \mathrm{H}^{n}(D)$ and $[\eta] \in \mathrm{H}^{m}(D)$, they decompose as $[\xi]=\left[\xi_{0}\right]+\left[\xi_{1}\right]$ and $[\eta]=\left[\eta_{0}\right]+\left[\eta_{1}\right]$ for cocycles $\xi_{0} \in D_{0}^{n}, \xi_{1} \in D_{\mathbb{R}}^{n}, \eta_{0} \in D_{0}^{m}$ and $\eta_{1} \in D_{\mathbb{R}}^{m}$. By definition, $\underline{\iota}^{*}\left(\left[\xi_{0}\right]\right)=\underline{\iota}^{*}\left(\left[\eta_{0}\right]\right)=0$ and since $\operatorname{ker}\left(\underline{\iota}^{*}\right)$ is an ideal we obtain

$$
\underline{\iota}^{*}([\xi] \smile[\eta])=\underline{\iota}^{*}\left(\left[\xi_{0}\right] \smile\left[\eta_{0}\right]+\left[\xi_{0}\right] \smile\left[\eta_{1}\right]+\left[\xi_{1}\right] \smile\left[\eta_{0}\right]+\left[\xi_{1}\right] \smile\left[\eta_{1}\right]\right)=\underline{\iota}^{*}\left(\left[\xi_{1}\right] \smile\left[\eta_{1}\right]\right)
$$

Since $\xi_{1}$ and $\eta_{1}$ take values in essentially constant functions on $Y$ we obtain

$$
\begin{aligned}
p_{2}^{*} \circ \underline{\iota}^{*}\left(\left[\xi_{1}\right] \smile\left[\eta_{1}\right]\right)\left(h_{0}, \ldots, h_{n+m+1}\right) & =\int_{Y}\left(\xi_{1} \smile \eta_{1}\right)\left(h_{0}, \ldots, h_{n+m+1}\right) \mathrm{d} \nu \\
& =\int_{Y} \xi_{1}\left(h_{0}, \ldots, h_{n}\right) \eta_{1}\left(h_{n}, \ldots, h_{n+m+1}\right) \mathrm{d} \nu \\
& =\int_{Y} \xi_{1}\left(h_{0}, \ldots, h_{n}\right) \mathrm{d} \nu \int_{Y} \eta_{1}\left(h_{n}, \ldots, h_{n+m+1}\right) \mathrm{d} \nu \\
& =p_{2}^{*} \circ \underline{\iota}^{*}\left(\left[\xi_{1}\right]\right) \smile p_{2}^{*} \circ \underline{\iota}^{*}\left(\left[\eta_{1}\right]\right)\left(h_{0}, \ldots, h_{n+m+1}\right)
\end{aligned}
$$

The composition $p^{*} \circ I_{0}^{*}: \mathrm{H}^{*}(G, \mathbb{R}) \rightarrow \underline{\mathrm{H}}^{*}(H, \mathbb{R})$ is therefore multiplicative and since we already argued, in the beginning of this section, that this map descends to an isomorphism $\underline{H}^{*}(G, \mathbb{R}) \simeq$ $\underline{\mathrm{H}}^{*}(H, \mathbb{R})$ it follows that it too is multiplicative.

Remark 4.5. Of course one could also define a cup product on $\mathrm{H}^{*}(G, \mathbb{C})$ and the arguments above obviously generalise to this setting so that the analogue of Theorem $\mathrm{B}$ over the complex numbers holds true as well.

\section{An APPLicAtion}

It is a well known open problem to classify connected simply connected (csc) nilpotent Lie groups up to quasi-isometry, and conjecturally quasi-isometry actually coincides with isomorphism on this class of groups [Cor18]. Although isomorphism classification is wide open in general, csc nilpotent Lie groups of dimension at most 7 are completely classified (up to isomorphism) by means of the corresponding classification of nilpotent real Lie algebras. This classification is the work by many hands, and we will here focus on Gong's thesis Gon98 which provides the first complete classification of all 7-dimensional, real, nilpotent Lie algebras (It should, however, be noted that Gra07] has pointed out that this classification is not complete in the 6-dimensional case). Regarding the quasi-isometry problem mentioned above, there are basically two main results supporting the conjecture.

Firstly, by Pansu's celebrated paper Pan89, if $G$ and $H$ are quasi-isometric csc nilpotent Lie groups with Lie algebras $\mathfrak{g}$ and $\mathfrak{h}$, respectively, then their associated Carnot Lie algebras $\mathfrak{C a r}(\mathfrak{g})$ and $\mathfrak{C} \mathfrak{a r}(\mathfrak{g})$ are isomorphic. Recall that if $\mathfrak{g}$ is a step $c$ nilpotent Lie algebra with lower 
central series $\mathfrak{g}=\mathfrak{g}_{1} \geqslant \mathfrak{g}_{2} \geqslant \ldots \geqslant \mathfrak{g}_{c} \geqslant\{0\}$, then its Carnot algebra is defined as

$$
\mathfrak{C a r}(\mathfrak{g}):=\bigoplus_{i=1}^{c} \mathfrak{g}_{i} / \mathfrak{g}_{i+1},
$$

with Lie bracket given, for $\bar{\xi} \in \mathfrak{g}_{i} / \mathfrak{g}_{i+1}$ and $\bar{\eta} \in \mathfrak{g}_{j} / \mathfrak{g}_{j+1}$, by $[\bar{\xi}, \bar{\eta}]:=\overline{[\xi, \eta]} \in \mathfrak{g}_{i+j} / \mathfrak{g}_{i+j+1}$. The Lie algebra $\mathfrak{g}$ is said to be Carnot, if $\mathfrak{g} \simeq \mathfrak{C a r}(\mathfrak{g})$, and from Pansu's theorem it therefore follows that if the csc nilpotent Lie groups associated with two Carnot, nilpotent Lie algebras are quasi-isometric, then they are in fact isomorphic.

Secondly, if $G$ and $H$ both contain lattices, i.e. admit rational structures Mal51, then by Sau06, Theorem 5.1] and [Nom54, Theorem 1] one may conclude that $\mathrm{H}^{*}(G, \mathbb{R})$ and $\mathrm{H}^{*}(H, \mathbb{R})$ are isomorphic as graded-commutative $\mathbb{R}$-algebras, and this also prevents many low dimensional csc nilpotent Lie groups from being quasi-isometric; see Cor18 for the state of the art in this direction.

Our Corollary C generalises the latter result (see Section 2.1.1 for this) and in the following sections we give examples showing how it can be used to distinguish new csc nilpotent Lie groups up to quasi-isometry. The key here is that for a csc nilpotent Lie group $G$ with Lie algebra $\mathfrak{g}$, the van Est theorem vE55 provides an isomorphism of graded-commutative $\mathbb{R}$ algebras $\mathrm{H}^{*}(G, \mathbb{R}) \simeq \mathrm{H}^{*}(\mathfrak{g}, \mathbb{R})$, where the latter denotes the Lie algebra cohomology of $\mathfrak{g}$ (see e.g. Gui80, Chapter II]). Computations of group cohomology may therefore be pushed to the Lie algebra side, and thus reduced to problems in finite dimensional linear algebra which can be solved by a computer.

5.1. Separation by Betti numbers. The aim of this section is to show how Corollary C can be used to give new examples of non-quasi-isometric, 7-dimensional, csc, nilpotent Lie groups. The main virtue of these examples is that the use of computer algebra systems needed is relatively light compared to the more advanced separation/non-separation results in the following section, which rely heavily on solving large systems of polynomial equations. Recall that Gong's list Gon98 contains six 1-parameter (where the parameter ranges over $\mathbb{C}$ with the possible exception of finitely many values) families of complex 7-dimensional nilpotent Lie algebras, and nine 1-parameter families (with parameter ranging over $\mathbb{R}$ with the possible exception of finitely many values) of real 7-dimensional nilpotent Lie algebras. To give the reader an idea of what these families look like we will present one example of a real 1-parameter family. We remark that we will use the notation used by Gong throughout this section, so that $\left\{x_{1}, \ldots, x_{7}\right\}$ will always denote the basis elements of a given Lie algebra, and $\langle\cdots\rangle$ is used to denote the $\mathbb{R}$-linear span of vectors. As is customary, all non-specified brackets between basis elements are implicitly set to zero.

1357M. The family $1357 M$ has the following bracket relations for $\lambda \in \mathbb{R} \backslash\{0\}$ :

$$
\begin{array}{lll}
{\left[x_{1}, x_{2}\right]=x_{3},} & {\left[x_{1}, x_{i}\right]=x_{i+2}, i=3,4,5,} & {\left[x_{2}, x_{4}\right]=x_{5},} \\
{\left[x_{2}, x_{6}\right]=\lambda x_{7},} & {\left[x_{3}, x_{4}\right]=(1-\lambda) x_{7} .}
\end{array}
$$

The family has lower central series

$$
\left\langle x_{1}, x_{2}, x_{3}, x_{4}, x_{5}, x_{6}, x_{7}\right\rangle \geqslant\left\langle x_{3}, x_{5}, x_{6}, x_{7}\right\rangle \geqslant\left\langle x_{5}, x_{7}\right\rangle \geqslant\left\langle x_{7}\right\rangle,
$$

and Carnot algebra given by the relations

$$
\left[\bar{x}_{1}, \bar{x}_{2}\right]=\bar{x}_{3}, \quad\left[\bar{x}_{1}, \bar{x}_{i}\right]=\bar{x}_{i+1}, i=3,4,5 .
$$


The Carnot algebra corresponds to the 7-dimensional real Lie algebra denoted by $2457 \mathrm{~A}$ in Gon98.

We may give a summary of the algebras, their lower central series dimensions and Betti numbers by means of the following table:

\begin{tabular}{|c|c|c|c|c|c|c|}
\hline $\mathbb{C}$ & $123457 I$ & $12457 N$ & $1357 N$ & $1357 S$ & $1357 M$ & $147 E$ \\
\hline $\mathbb{R}$ & $123457 I$ & \begin{tabular}{l|l}
$12457 N$ & $12457 N_{2}$ \\
\end{tabular} & $1357 N$ & \begin{tabular}{l|l}
$1357 S$ & $1357 Q R S_{1}$ \\
\end{tabular} & $1357 M$ & \begin{tabular}{l|l}
$147 E$ & $147 E_{1}$ \\
\end{tabular} \\
\hline$\beta^{n}$ & 12344321 & 12344321 & 13577531 & 13677631 & 13688631 & 13799731 \\
\hline $\mathrm{LC}$ & 754321 & 75421 & 7421 & 7431 & 7421 & 741 \\
\hline $\mathfrak{C a r}(\mathfrak{g})$ & $123457 A$ & \begin{tabular}{l|l}
$12457 L$ & $12457 L_{1}$
\end{tabular} & $2457 A$ & See below & $2457 A$ & \begin{tabular}{l|l}
$147 E$ & $147 E_{1}$
\end{tabular} \\
\hline
\end{tabular}

TABLE 1. Summary of 1-parameter families of 7-dimensional nilpotent Lie algebras.

The first row consists of the the complex families, the second of the real families (the complexification of each being the algebra right above it), and the third consists of the sequence of Betti numbers; i.e. the dimensions of the real cohomology groups. The fourth row consists of the dimensions of the algebras appearing in the lower central series, and the fifth row contains the Carnot algebra of the family in question. Here the families and their complexifications may be found listed in Gon98, and the Betti numbers and lower central series dimensions can easily be computed in e.g. Maple. We note that Mag08 also provides a complete list of Betti numbers from which these may also be taken. We stress that we are here only using the generic Betti numbers: as one can see from e.g. Magnin's paper, several families have a finite number of parameter-values for which the Betti numbers are different from the ones listed, but since these finitely many non-generic cases are irrelevant for our purposes, we will disregard them here. From the lower central series, the Carnot algebras are easily computed and may be compared (e.g. using Maple) with those found in Gong's list. For most families one obtains a single (family of) Carnot algebra(s), but for the families $1357 S$ and $1357 Q R S_{1}$ the situation is not quite as simple: one here obtains that the family $1257 S$ (with parameter $\lambda \in \mathbb{R} \backslash\{1\}$ ) has Carnot algebra $N_{6,2,5} \oplus \mathbb{R}$ when $\lambda<1$ and $N_{6,2,5 a} \oplus \mathbb{R}$ when $\lambda>1$. Similarly, $1357 Q R S_{1}$ (with parameter $\lambda \in \mathbb{R} \backslash\{0\}$ ) has associated Carnot algebra $N_{6,2,5} \oplus \mathbb{R}$ when $\lambda<0$ and $N_{6,2,5 a} \oplus \mathbb{R}$ when $\lambda>0$.

We now turn towards the quasi-isometry classification problem for members of different families. By Pansu's theorem, the Carnot algebra is a quasi-isometry invariant, so in most cases Table 5.1 rules out the possibility that the csc nilpotent Lie groups associated with two members of different families can be quasi-isometric. However, the families $1357 \mathrm{~N}$ and $1357 \mathrm{M}$ are seen to have the same associated Carnot Lie algebra and hence cannot be separated by Pansu's theorem, but they do have different Betti numbers, and thus separation here follows from Corollary C. Since at most a countable number of members of each family have an associated csc Lie group which admits a lattice Mal51, this illustrates an instance where Corollary C can tell apart (up to quasi-isometry) Lie groups that are not distinguished by neither the results of Pansu, nor by those of Sauer and Shalom [Sau06, Sha04. We note that $1357 S$ and $1357 Q R S_{1}$ are the only ones for which no generic separation between the two families can be made from neither Pansu's theorem nor their Betti numbers, although Pansu's 
theorem of course proves partial information, in that members whose parameter values give rise non-isomorphic Carnot algebras cannot be quasi-isometric.

5.2. Separation within continuous 1-parameter families. Another natural question is whether it is possible to use Corollary $\mathrm{C}$ to find the first uncountable family of pairwise nonquasi-isometric csc nilpotent Lie groups that are not already distinguished by Pansu's teorem [Pan89, Théorème 3]. As can be seen from Table 5.1, the nine 1-parameter families of real 7-dimensional nilpotent Lie algebras fall in three groups:

(i) $147 E$ and $147 E_{1}$ which are Carnot;

(ii) $123457 I, 12457 \mathrm{~N}, 12457 \mathrm{~N}_{2}, 1357 \mathrm{M}$ and $1357 \mathrm{~N}$ which are not Carnot, but within each family the associated family of Carnot algebras, generically, consists of isomorphic members;

(iii) $1357 S$ and $1357 Q R S_{1}$, where each family has two isomorphism classes of associated Carnot algebras.

We will now show that each family in the second class (ii) have generically isomorphic cohomology algebras, and that each family in the third class (iii) gives rise to cohomology algebras which fall within uncountably many isomorphism classes. For results about the class (i), see Remark 5.5.

Using Maple, one can compute a linear basis for the cohomology of any given finite dimensional Lie algebra, as well as multiplication tables for for the cup product. As an illustration of the type of multiplication tables one encounters when doing this for the 1-paramter families, we include here two examples:

\begin{tabular}{|cccccccc|}
\hline 0 & 0 & $-\frac{t^{4}-2 t^{3}+4 t^{2}-2 t+2}{t^{2}-t+1}$ & 0 & 0 & 0 & 0 & $-\frac{t^{2}+2}{t^{2}-t+1}$ \\
0 & $-\frac{6 t^{4}-6 t^{3}+13 t^{2}-4 t+6}{t^{2}\left(t^{2}-4 t+4\right)}$ & 0 & 0 & 0 & 0 & 0 & 0 \\
$-\frac{t^{2}-2 t+2}{t^{2}-2 t+1}$ & 0 & 0 & 0 & 0 & 0 & 0 & 0 \\
0 & 0 & $-\frac{t^{3}-3 t^{2}+4 t-2}{t^{2}-t+1}$ & 0 & 0 & 0 & 0 & $-\frac{t^{2}+2}{t^{2}-t+1}$ \\
0 & 0 & 0 & 0 & 0 & 0 & $2 \frac{t^{2}+t+1}{t^{2}+2 t+1}$ & 0 \\
0 & 0 & 0 & 0 & 0 & -1 & 0 & 0 \\
0 & 0 & 0 & 0 & $\frac{t^{4}-2 t^{3}+5 t^{2}-4 t+6}{t^{4}-2 t^{3}+3 t^{2}-2 t+1}$ & 0 & 0 & 0 \\
0 & 0 & 0 & 1 & 0 & 0 & 0 & 0 \\
\hline
\end{tabular}

TABLE 2. Multiplication table for 3rd and 4th cohomology for the family $1357 M$

The tables should be read as follows: the parameter $t$ is the parameter of the family $\left(\mathfrak{g}_{t}\right)_{t}$ in question. In Table 1 , we have that $\mathrm{H}^{3}(\mathfrak{g}(t), \mathbb{R})$ is 8 dimensional with a fixed basis $e_{3}^{1}(t), \ldots, e_{3}^{8}(t)$, that $\mathrm{H}^{4}(\mathfrak{g}(t), \mathbb{R})$ is also 8 dimensional with a fixed basis $e_{4}^{1}(t), \ldots, e_{4}^{8}(t)$ and that $\mathrm{H}^{7}(\mathfrak{g}(t), \mathbb{R})$ is one dimensional with a fixed basis $e_{7}(t)$. The $(i, j)$ th entry of the table is the coefficient of the product $e_{3}^{i}(t) \smile e_{4}^{j}(t)$; e.g. we have

$$
e_{3}^{1}(t) \smile e_{4}^{3}(t)=-\frac{t^{4}-2 t^{3}+4 t^{2}-2 t+2}{t^{2}-t+1} e_{7}(t)
$$

from the first table. A similar reasoning applies to Table 2 . 


\begin{tabular}{|cccc|}
\hline$-\frac{3 t^{4}-3 t^{3}+32 t^{2}-10 t+108}{3 t\left(t^{2}-2 t+6\right)}-\frac{3 t^{6}-6 t^{5}+50 t^{4}-52 t^{3}+276 t^{2}-120 t+504}{6 t\left(t^{2}-2 t+6\right)}-\frac{15 t^{4}-30 t^{3}+158 t^{2}-160 t+432}{3 t\left(t^{2}-2 t+6\right)}$ & $\frac{3 t^{5}+6 t^{4}+14 t^{3}+100 t^{2}-28 t+360}{6 t\left(t^{2}-2 t+6\right)}$ \\
$\frac{3 t^{3}+2 t^{2}+7 t+8}{2\left(t^{2}-2 t+6\right)}$ & $\frac{3 t^{6}+5 t^{5}+27 t^{4}+37 t^{3}+78 t^{2}+42 t+48}{4\left(t^{3}-t^{2}+4 t+6\right)}$ & $-\frac{3 t^{4}+5 t^{3}+5 t^{2}+23 t-16}{t^{3}-t^{2}+4 t+6}$ & $-\frac{3 t^{5}-7 t^{4}-t^{3}-45 t^{2}-34 t-196}{4\left(t^{3}-t^{2}+4 t+6\right)}$ \\
$\frac{11 t^{2}-2 t+41}{3\left(t^{2}-2 t+6\right)}$ & $\frac{11 t^{4}-2 t^{3}+107 t^{2}-12 t+246}{6\left(t^{2}-2 t+6\right)}$ & $-\frac{2\left(11 t^{2}-2 t+41\right)}{3\left(t^{2}-2 t+6\right)}$ & $-\frac{11 t^{3}-24 t^{2}+45 t-82}{6\left(t^{2}-2 t+6\right)}$ \\
$-\frac{3 t^{3}-14 t^{2}+28 t-27}{3 t\left(t^{2}-2 t+6\right)}$ & $\frac{8 t^{4}-4 t^{3}+57 t^{2}-12 t+126}{6 t\left(t^{2}-2 t+6\right)}$ & $\frac{11 t^{2}-34 t+108}{3 t\left(t^{2}-2 t+6\right)}$ & $-\frac{8 t^{3}-2 t^{2}-t+90}{6 t\left(t^{2}-2 t+6\right)}$ \\
\hline
\end{tabular}

TABLE 3. Multiplication table for 3rd and 4th cohomology for the family $12457 \mathrm{~N}$

We now explain how to construct isomorphisms of graded $\mathbb{R}$-algebras

$$
\varphi_{t, s}: \mathrm{H}^{*}(\mathfrak{g}(s), \mathbb{R}) \rightarrow \mathrm{H}^{*}(\mathfrak{g}(t), \mathbb{R}) .
$$

Denote again the fixed basis of $\mathrm{H}^{*}(\mathfrak{g}(\lambda), \mathbb{R})$ by $e_{i}^{j}(\lambda)$, so that for fixed $i$ the elements $e_{i}^{j}(\lambda)$ is a basis for $\mathrm{H}^{i}(\mathfrak{g}(\lambda), \mathbb{R})$. Using the fact that $\varphi_{t, s}$ should be linear and graded we know that $\varphi_{t, s}$ is given by the coefficients associated with the image of the basis elements:

$$
\varphi_{t, s}\left(e_{i}^{j}(s)\right)=\sum_{k=1}^{\beta_{i}(\mathfrak{g}(t))} \alpha_{i, j}^{k} e_{i}^{k}(t) .
$$

In other words, we may consider $\varphi$ as a block-diagonal matrix, where the blocks are $\beta_{i}(\mathfrak{g}(t)) \times$ $\beta_{i}(\mathfrak{g}(t))$-matrices. The map $\varphi$ being an $\mathbb{R}$-algebra homomorphism is then encoded by solutions to a polynomial system of equations in the variables $\alpha_{i, j}^{k}$ arising from the multiplication table, and one can easily check whether $\varphi$ is bijective or not by taking the determinant of the matrix corresponding to the solutions.

The method used to obtain solutions to the system is as follows, and the implementation has been done in Maple 19: All equations appearing are polynomial in the variables $\alpha_{i, j}^{k}$ with certain rational functions in the parameters $s$ or $t$ as coefficients. First the system is simplified by inspecting the equations, and replacing every equation of the form $p(s) \alpha_{i, j}^{k}=0$ (with $p$ a rational function in $s$ ) with $\alpha_{i, j}^{k}=0$, and similarly for equations of the form $p(t) \alpha_{i, j}^{k} \alpha_{\ell, m}^{n}=0$. Then using the zero-product rule on equations of the second type above, along with a nonsingularity assumption on all the block-matrices along the diagonal, one may in certain cases conclude which factor should be 0 . To illustrate this, consider the family $1357 M$ which has $\beta_{1}(\mathfrak{g}(t))=3$ for all but finitely many values of $t$, and for which the corresponding system of equations contains the following three:

$$
\alpha_{1,1}^{1} \alpha_{4,4}^{6}=0, \quad \alpha_{1,2}^{1} \alpha_{4,4}^{6}=0, \quad \alpha_{1,3}^{1} \alpha_{4,4}^{6}=0
$$

Since $\alpha_{1,1}^{1}=0, \alpha_{1,2}^{1}=0$ and $\alpha_{1,3}^{1}=0$ would cause the first block-matrix to be singular, we may conclude that $\alpha_{4,4}^{6}=0$. These zero-values are then substituted into the system which is then reduced. The procedure is then continued until no further conclusions can be made this way. After this, we tried solving the system. In case the system was still too complex to solve in reasonable time, we would make qualified guesses on values of variables based on solutions of parts of the system. This process could be repeated several times. After a full solution was obtained, we would set any remaining free variables to 1 , compute the determinants of the diagonal matrices, and verify the solution by substituting it into the system. For all families 
of the second class (ii), this provided a solution which was an isomorphism for all but finitely many values of the parameter, so that we have the following:

Proposition 5.1. Within each of the 7-dimensional one-parameter families 123457 I, $12457 \mathrm{~N}$, $12457 N_{2}, 1357 M$ and $1357 N$ of nilpotent Lie algebras, all members, except for perhaps finitely many, have isomorphic cohomology algebras.

Thus, Corollary C cannot be used to tell the Lie groups associated with the members of these families apart up to quasi-isometry. We remark that obtaining solutions to the equation systems described above carries a high degree of complexity. For example, the system of equations for the family 1357M consists of 1193 unique (but possibly equivalent) equations, with up to 11 summands of the form $p(t) \alpha_{j, i}^{k} \alpha_{\ell, m}^{n}$, where the coefficients $p(t)$, as already mentioned, are rational functions in $t$. In contrast to this, the system obtained from the family $12457 N$ consists of 94 equations, each of which contains up to 17 summands. The concrete formulas for the graded $\mathbb{R}$-algebra isomorphisms $\varphi_{t, s}: \mathrm{H}^{*}(\mathfrak{g}(s), \mathbb{R}) \rightarrow \mathrm{H}^{*}(\mathfrak{g}(t), \mathbb{R})$ are not included here due to their sizes; for instance for the family $12457 N$ the $\varphi_{t, s}$ is given by $20 \times 20$-matrix, and the expression below is an example of one of its prototypical non-zero entries:

$$
\begin{aligned}
& \frac{3(t-s)}{(t+1)\left(6 t^{3}-19 t^{2}-16 t-167\right)(s+1)\left(s^{3}-2 s^{2}+17 s-2\right)}\left[\left(-4+t^{4}-\frac{7}{6} t^{3}-\frac{20}{3} t^{2}+\frac{37}{6} t\right) s^{4}+\right. \\
+ & \left(2-\frac{7}{6} t^{4}-\frac{62}{3} t^{3}-\frac{143}{6} t^{2}-17 t\right) s^{3}+\left(\frac{207}{2} t-\frac{175}{3}-\frac{20}{3} t^{4}-\frac{143}{6} t^{3}-\frac{226}{3} t^{2}\right) s^{2}+ \\
+ & \left.\left(-\frac{400}{3}+\frac{37}{6} t^{4}-17 t^{3}+\frac{207}{2} t^{2}+\frac{640}{3} t\right) s-\frac{400}{3} t-4 t^{4}+2 t^{3}-\frac{119}{3}-\frac{175}{3} t^{2}\right]
\end{aligned}
$$

Maple worksheets performing this procedure with full solutions (and the correct guesses written down) can be obtained from the authors upon request; see also Remark 5.6.

For the two families in (iii), we will now show that the picture is quite different, in that the associated families of cohomology algebras fall into uncountably many isomorphism classes. As noted earlier, the families are not separable by Pansu's theorem, as it can see only two quasi-isometry classes. However, by appealing to Corollary C we do in fact obtain uncountably many classes:

Theorem 5.1. Let $(\mathfrak{g}(\lambda))_{\lambda}$ denote either of the families $1357 S$ and $1357 Q R S_{1}$. Then for every parameter value $\lambda_{0}$ there exist at most finitely many other parameter values $\lambda$ for which $\mathrm{H}^{*}(\mathfrak{g}(\lambda), \mathbb{R}) \simeq \mathrm{H}^{*}\left(\mathfrak{g}\left(\lambda_{0}\right), \mathbb{R}\right)$. In particular, the 1-parameter families of csc nilpotent Lie groups associated with $1357 S$ and $1357 Q R S_{1}$ fall into uncountably many quasi-isometry classes.

To prove Theorem [5.1, one could of course again implement the equations satisfied by a potential isomorphism in Maple with the hope that there are no solutions. However, doing so did not lead to any conclusion as an attempt to solve the system did not terminate within a reasonable time frame, and any guesses added to the system would make it unable to be used to disprove the existence of isomorphisms. Instead, we will require a bit of deformation theory to prove Theorem 5.1. For our purpose a very ad hoc introduction suffices, but the interested reader may consult [Ger64] for a more thorough presentation.

Given an algebra $A$, a one-parameter (formal) deformation of $A$, is a formal power series $F=\sum_{i=0}^{\infty} f_{i} t^{i}$ such that the coefficients are linear maps $f_{i}: A \otimes A \rightarrow A$ and $f_{0}$ is the multiplication in $A$. The first nontrivial $f_{i}$ with $i \geqslant 1$ is called the infinitesimal of the deformation. The power series $F$ defines a product on the space of formal power series $A[[t]]$ 
with coefficients in $A$, by defining $a * b:=\sum_{i=0}^{\infty} f_{i}(a, b) t^{i}$ for $a, b \in A$ and extending to all of $A[[t]]$. If this product is associative, the infinitesimal $f_{i_{0}}: A \otimes A \rightarrow A$ becomes a Hocschild 2-cocycle. There is of course a notion of triviality and equivalence between deformations, and these concepts turn out to be intimately linked with triviality of the class of $f_{i_{0}}$ in $\mathrm{H}^{2}(A, A)$ (see e.g. Ger64). To this end, we recall that $f_{i_{0}}$ is a Hochschild coboundary if there exists a linear map $g: A \rightarrow A$ such that $f_{i_{0}}=\partial_{1} g$, where

$$
\partial_{1}(g)(a, b):=a g(b)-g(a b)+g(a) b .
$$

If the algebra $A$ comes equipped with a topology and $\lambda \in \mathbb{R}$ is such that the series $F_{t}(a, b)=$ $\sum_{i=0}^{\infty} f_{i}(a, b) t^{i}$ converges for $t=\lambda$, then $\left(A, F_{\lambda}\right)$ becomes an algebra (associative if $F$ is so) called the specialisation of $F$ at $\lambda$. In general, a (formal) deformation may not have any specialisations, but we will show below that when $\left(\mathfrak{g}_{\lambda}\right)_{\lambda}$ is one of the families $1357 S$ or $1357 Q R S_{1}$, then the family of cohomology algebras $\left(\mathrm{H}^{*}\left(\mathfrak{g}_{\lambda}, \mathbb{R}\right)\right)_{\lambda}$ may be viewed as specialisations of a formal deformation. We are of course interested in whether or not the cohomology algebras $\left(H^{*}\left(\mathfrak{g}_{\lambda}, \mathbb{R}\right)\right)_{\lambda}$ are pairwise isomorphic, which is an a priori different notion of triviality than the one considered in formal deformation theory, but as the following two lemmas will show, triviality/non-triviality of the 2 -cocycle $f_{1}$, which turns out to be the infinitesimal, again plays an important role.

Lemma 5.2. Let $A$ be a finite-dimensional real algebra, equipped with the Euclidean topology, and let $F$ be a deformation of $A$. Assume that there is a neighbourhood of zero, $U \subset$ $\mathbb{R}$, on which $F$ admits specialisations $\left\{A_{\lambda}\right\}_{\lambda \in U}$, such that there exists a family of isomorphisms $\left\{\varphi_{\lambda}: A_{\lambda} \rightarrow A_{0}\right\}_{\lambda \in U}$ with $\varphi_{0}$ being the identity. Assume further that $\left.\frac{d}{d \lambda} \varphi_{\lambda}(a)\right|_{\lambda=0}:=$ $\lim _{\lambda \rightarrow 0} \frac{\varphi_{\lambda}(a)-a}{\lambda}$ exists for all $a \in A$. Then the map $g: A \rightarrow A$ defined by $g(a)=\left.\frac{d}{d \lambda} \varphi_{\lambda}(a)\right|_{\lambda=0}$ satisfies $\partial_{1} g=f_{1}$; thus $f_{1}$ is a Hochschild coboundary.

Proof. This is an easy consequence of taking the derivative at zero on both sides of the equation $\varphi_{\lambda}(a) \varphi_{\lambda}(b)=\varphi_{\lambda}(a b)+\sum_{k=1}^{\infty} \varphi_{\lambda}\left(f_{i}(a, b)\right) \lambda^{i}$, noting that $\varphi_{0}$ is the identity so that the Leibniz rule gives

$$
\left.\frac{d}{d \lambda}\left(\varphi_{\lambda}(a) \varphi_{\lambda}(b)\right)\right|_{\lambda=0}=a g(b)+g(a) b
$$

and that

$$
\left.\frac{d}{d \lambda} \varphi_{\lambda}\left(f_{i}(a b) \lambda^{i}\right)\right|_{\lambda=0}=\left\{\begin{array}{cc}
g(a b) & i=0 \\
f_{1}(a, b) & i=1 \\
0 & i>1
\end{array}\right.
$$

Lemma 5.3. Let $V$ be a finite dimensional vector space with a fixed basis $e_{1}, \ldots, e_{n}$, and let $U \subset \mathbb{R}$ be an open interval such that for each $\lambda \in U$ one has a product $*_{\lambda}$ on $V$ turning it into an associative real algebra $A_{\lambda}$. Assume moreover that the coefficients of $e_{k} *_{\lambda} e_{l}$ relative to the fixed basis are given by rational functions in $\lambda$. Fix $\lambda_{0} \in U$ and assume that for infinitely many $\lambda \in U$ there exists an algebra-isomorphism from $A_{\lambda}$ to $A_{\lambda_{0}}$. Then there exists an open set $V \subset U$ and $n \times n$-matrices $\left(\left(\alpha_{i j}(\lambda)\right)_{i j}\right)_{\lambda \in V}$ such the associated maps in $\operatorname{End}(V)$ are algebraisomorphisms $A_{\lambda} \simeq A_{\lambda_{0}}$ and such that $\lambda \mapsto \alpha_{i j}(\lambda)$ is differentiable for every $1 \leqslant i, j \leqslant n$.

The lemma is a consequence of the so-called Nash cell decomposition of semi-algebraic sets; we refer to Chapter 2 of [BCR98] for the basics of the this theory. 
Proof. Denote by $S \subset \mathbb{R}^{n^{2}+1}$ the set of $\left(\alpha_{i j}(\lambda), \lambda\right)$ where $\lambda \in U$ and $\left(\alpha_{i j}(\lambda)\right)_{i, j} \in \mathbb{M}_{n}(\mathbb{R})$ implements and algebra-isomorphism $A_{\lambda} \simeq A_{\lambda_{0}}$. Since the scalar coefficients of products are rational functions in $\lambda$, the system of equations which encodes that $\left(\alpha_{i j}(\lambda)\right)_{i j}$ is an algebraisomorphism consists of polynomials in $\alpha_{i j}(\lambda)$ and $\lambda$, inequations of the form $\lambda \neq x$ where $x$ is a root in a polynomial appearing in a denominator of one of the rational functions, and the polynomial inequation $\operatorname{det}\left(\left(\alpha_{i j}(\lambda)\right)_{i j}\right) \neq 0$. Since $U$ is an interval, the set $S$ is therefore a semi-algebraic set in $\mathbb{R}^{n^{2}+1}$ (see [BCR98] for the relevant definitions), and by [BCR98, Proposition 2.9.10], $S$ admits a decomposition into a disjoint union of finitely many submanifolds

$$
S=A_{1} \sqcup \cdots \sqcup A_{n}
$$

such that each $A_{i}$ is either a singleton set or diffeomorphic to an open hypercube $(0,1)^{d_{i}} \subset$ $\mathbb{R}^{d_{i}}$. Since we assume that solutions exist for infinitely many $\lambda \in U$, we may, without loss of generality, assume that $A_{1}$ contains at least two points, $p_{1}:=\left(\left(\alpha_{i j}\left(\lambda_{1}\right)\right)_{i j}, \lambda_{1}\right)$ and $p_{2}:=\left(\left(\alpha_{i j}\left(\lambda_{2}\right)\right)_{i j}, \lambda_{2}\right)$, with $\lambda_{1}<\lambda_{2}$. Let now $\varphi: A_{1} \rightarrow(0,1)^{d_{1}}$ be a diffeomorphism and let $\gamma:[0,1] \rightarrow(0,1)^{d_{1}}$ be the straight line segment between $\varphi\left(p_{1}\right)$ and $\varphi\left(p_{2}\right)$, and let $\pi_{n^{2}+1}: \mathbb{R}^{n^{2}+1} \rightarrow \mathbb{R}$ denote the projection onto the last coordinate. Then the map $f:[0,1] \rightarrow \mathbb{R}$ given by $f(t)=\pi_{n^{2}+1} \circ \varphi^{-1} \circ \gamma$ is continuous and differentiable on $(0,1)$ and satisfies $f(0)=\lambda_{1}$ and $f(1)=\lambda_{2}$. Hence there is a $\hat{t} \in(0,1)$ such that $f^{\prime}(\hat{t}) \neq 0$, and thus $f$ is invertible on a neighbourhood of $\hat{t}$. On an open interval $I$ around $\hat{\lambda}:=f(\hat{t})$, we may therefore consider $\psi=\varphi^{-1} \circ \gamma \circ f^{-1}: I \rightarrow \mathbb{R}^{n^{2}+1}$ and we have that $\left(\alpha_{i j}(\lambda), \lambda\right):=(\psi(\lambda))$ is a solution, and each $\alpha_{i j}(\lambda)$ is differentiable.

We now return to the concrete algebras we wish to handle, namely the cohomology algebras arising from the 1-parameter families $1357 S$ and $1357 Q R S_{1}$. In each case, using Maple one obtains a basis for the total cohomology $\mathrm{H}^{*}\left(\mathfrak{g}_{\lambda}, \mathbb{R}\right)$ and multiplication tables for the cup product, in which all the coefficients are rational functions in $\lambda$. For all but finitely many parameter values the vector space $\mathrm{H}^{*}\left(\mathfrak{g}_{\lambda}, \mathbb{R}\right)$ has a fixed dimension, and for these parameter values we may therefore consider the algebras $\mathrm{H}^{*}\left(\mathfrak{g}_{\lambda}, \mathbb{R}\right)$ as a 1-parameter family of products $\smile_{\lambda}$ on a fixed vector space $V$ with a fixed basis $e_{1}, \ldots, e_{n}$, thus putting us within the scope of Lemma 5.3. Denote by $p_{i j}^{k}$ the rational functions describing the product of $e_{i}$ and $e_{j}$; i.e.

$$
e_{i} \underset{\lambda}{\smile} e_{j}=\sum_{k=1}^{n} p_{i j}^{k}(\lambda) e_{k} .
$$

Since the coefficients are rational functions, they admit a power series expansion for all but finitely many $\lambda$, so we may construct a deformation around a chosen, nonsingular, $\lambda_{0}$ by shifting the variable and using the Maclaurin expansion; i.e. by setting

$$
f_{m}\left(e_{i}, e_{j}\right):=\left.\sum_{k=1}^{n} \frac{d^{m}}{d t^{m}} p_{i j}^{k}\right|_{t=\lambda_{0}} \frac{1}{m !} e_{k}
$$

we obtain a formal power series $F(a, b)=\sum_{m=0}^{\infty} f_{m}(a, b) t^{m}$ which converges for $t$ in a neighbourhood of zero and such that the specialisation at $t=0$ is exactly $\mathrm{H}^{*}\left(\mathfrak{g}_{\lambda_{0}}, \mathbb{R}\right)$. Thus, $F$ constitutes a (formal) deformation of the algebra $\mathrm{H}^{*}\left(\mathfrak{g}_{\lambda_{0}}, \mathbb{R}\right)$, and implementing the above in Maple, it turns out that for the two families in question, $f_{1}$ is non-zero for all but finitely many values of $\lambda_{0}$, and thus the infinitesimal here. 
In the light of Lemma 5.2, we now need to investigate if $f_{1}$ is a coboundary in each of the two cases, i.e. if there exists a linear map $g: \mathrm{H}^{*}\left(\mathfrak{g}\left(\lambda_{0}\right), \mathbb{R}\right) \rightarrow \mathrm{H}^{*}\left(\mathfrak{g}\left(\lambda_{0}\right), \mathbb{R}\right)$ such that

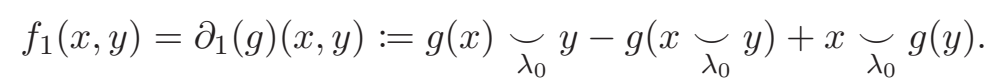

If such a map exists, it is uniquely determined by the scalars $b_{i j}$ satisfying

$$
g\left(e_{i}\right)=\sum_{j=1}^{n} b_{i j} e_{j},
$$

and setting $x=e_{i}$ and $y=e_{j}$ in (10) yields

$$
\begin{aligned}
\left.\sum_{k=1}^{n} \frac{d}{d t} p_{i j}^{k}\right|_{t=\lambda_{0}} e_{k} & =\sum_{k=1}^{n} b_{i k} e_{k} \underset{\lambda_{0}}{\smile} e_{j}-g\left(\sum_{k=1}^{n} p_{i j}^{k}\left(\lambda_{0}\right) e_{k}\right)+\sum_{k=1}^{n} b_{j k} e_{i} \smile_{\lambda_{0}} e_{k} \\
& =\sum_{k, l=1}^{n} b_{i k} p_{k j}^{l}\left(\lambda_{0}\right) e_{l}-\sum_{k, l=1}^{n} p_{i j}^{k}\left(\lambda_{0}\right) b_{k l} e_{l}+\sum_{k, l=1}^{n} b_{j k} p_{i k}^{l}\left(\lambda_{0}\right) e_{l} .
\end{aligned}
$$

Comparing coefficients of the basis vectors now gives a system of linear equations in the variables $b_{i j}$ and, by construction, $f_{1}$ is a Hochschild 1-coboundary if and only if this system has a solution. Both of the families $1357 S$ and $1357 Q R S_{1}$ have 34-dimensional cohomology algebras, and the resulting linear systems are much bigger than what can reasonably be solved by hand (the systems contain, respectively, 7066 and 6388 equations), but using Maple, it has been verified, that for all but a most finitely many values of $\lambda_{0}$ the corresponding system of equations does not have any solutions.

Proof of Theorem 5.1. Let $\left(\mathfrak{g}_{\lambda}\right)_{\lambda}$ be either of the two families. For all but finitely many parameter values, $\mathrm{H}^{*}\left(\mathfrak{g}_{\lambda}, \mathbb{R}\right)$ has a fixed dimension, so let $\lambda_{0}$ be such a generic parameter value and choose an open interval $U$ around $\lambda_{0}$ consisting of generic parameter values. As argued in the beginning of this section, we may then consider the algebras $\left(H^{*}\left(\mathfrak{g}_{\lambda}, \mathbb{R}\right)\right)_{\lambda \in U}$ as a family of algebra structures on a fixed vector space with a fixed basis, with the property that the coefficients of products of basis vectors are given by rational functions in $\lambda$. So, if $H^{*}\left(\mathfrak{g}_{\lambda_{0}}, \mathbb{R}\right) \simeq \mathrm{H}^{*}\left(\mathfrak{g}_{\lambda}, \mathbb{R}\right)$ for infinitely many other parameter values, then Lemma 5.3 provides a $\hat{\lambda}$, an open neighbourhood $V$ around $\hat{\lambda}$ and an isomorphism $\varphi_{\lambda}=\left(\alpha_{i j}(\lambda)\right)_{i j}: \mathrm{H}^{*}\left(\mathfrak{g}_{\lambda}, \mathbb{R}\right) \rightarrow \mathrm{H}^{*}\left(\mathfrak{g}_{\lambda_{0}}, \mathbb{R}\right)$ for each $\lambda \in V$ such that every $\alpha_{i j}(\lambda): U \rightarrow \mathbb{R}$ is differentiable. We remark that since $\operatorname{dim}_{\mathbb{R}} \mathrm{H}^{*}\left(\mathfrak{g}_{\lambda}, \mathbb{R}\right)$ only takes finitely many values, and since we constructed a formal deformation of $\mathrm{H}^{*}\left(\mathfrak{g}_{\lambda_{0}}, \mathbb{R}\right)$ for all but finitely many $\lambda_{0}$, and that the associated infinitesimals were shown to be Hochschild 1-coboundaries for only finitely many $\lambda_{0}$, we may therefore assume that $V$ does not intersect any of these finite sets of parameter values. Upon replacing $\varphi_{\lambda}$ with $\varphi_{\lambda} \circ \varphi_{\hat{\lambda}}^{-1}$ we may assume that $\varphi_{\hat{\lambda}}$ is the identity. Considering $\left(\mathrm{H}^{*}\left(\mathfrak{g}_{\lambda}, \mathbb{R}\right)\right)_{\lambda \in V}$ as specialisations of a formal deformation $F(-,-)=\sum_{k=0}^{\infty} f_{k}(-,-) t^{k}$ of $\mathrm{H}^{*}\left(\mathfrak{g}_{\hat{\lambda}}, \mathbb{R}\right)$ as described above, Lemma 5.2 now implies that the first term $f_{1}$ is a Hochschild 1-coboundary, contradicting what what was established in the paragraph preceding the proof.

Remark 5.4. We remark that none of the results related to Theorem 5.1 involve graded algebras or maps, but that we in fact show that no uncountable subfamily whose cohomology algebras are isomorphic, as algebras, exists. Since graded isomorphisms are also encoded by a system of polynomial equations, the proof of Lemma 5.3 carries over verbatim to the obvious graded restatement, but this has not been relevant for our purposes. 
Remark 5.5. As pointed out by an anonymous referee, it would be of interest to find two non-isomorphic Carnot Lie algebras, which have isomorphic cohomology algebras, thus providing examples of csc nilpotent Lie groups that are distinguished by Pansu's result, but not by their associated cohomology algebras. One such example occurs when considering the family 147E of class (i). Since the family is Carnot, the associated Lie groups can be distinguished by Pansu's result, and the method used to compute isomorphisms for Proposition 5.1 yields a graded isomorphism between the cohomology of all but finitely many members of the family, thus providing such an example.

Performing a similar check for the five (finite) families of Carnot Lie algebras of dimension at most 6, whose members share the same Betti numbers, shows that such a result cannot be obtained in dimension lower than seven. In all cases but for those Lie algebras which have the same complexification, this can be seen directly by comparing the ranks of the multiplication maps. For the pairs which share their complexification, it turns out that the associated system of equations cannot have any non-singular solution.

We have also attempted to check whether or not the cohomology algebras associated to the last remaining family, $147 \mathrm{E}_{1}$, are generically isomorphic, but this has been inconclusive. The method used to prove non-isomorphism for the algebras in class (iii), shows that the infinitesimals of the associated formal deformations are (generically) coboundaries, and hence we cannot conclude that the algebras are not isomorphic. On the other hand, all attempts to find a solution to the system of equations arising when applying the method used to prove isomorphism in cohomology for the families in class (ii) have thus far been inconclusive, since we could not reduce the system to a point where no guesses have to be made, and any combination of guesses we have tried have caused the resulting system to have no solutions. However, as pointed out by an anonymous referee, it is actually possible to give a complete Hochschild cohomological characterisation of when all (but finitely many) members of a family $\mathrm{H}^{*}\left(\mathfrak{g}_{\lambda}, \mathbb{R}\right)$ are pairwise isomorphic or not, which is likely to provide a way of settling this remaining issue, and also provide an alternative way of proving Proposition 5.1 requiring less computational effort, since one would have to consider a system of linear equations rather than a system of polynomial equations. However, this is beyond the scope of the present section, but we are grateful to the referee for providing us with this perspective on the problem.

Remark 5.6. Maple worksheets verifying the computer assisted claims made above can be obtained from the authors upon request or, at the time of writing, be downloaded via www.imada.sdu.dk/ thgot/

\section{REFERENCES}

[BFS13] Uri Bader, Alex Furman, and Roman Sauer. Integrable measure equivalence and rigidity of hyperbolic lattices. Invent. Math., 194(2):313-379, 2013.

[BHI18] Lewis Bowen, Daniel Hoff, and Adrian Ioana. von Neumann's problem and extensions of nonamenable equivalence relations. Groups Geom. Dyn., 12(2):399-448, 2018.

[Bla79] Philippe Blanc. Sur la cohomologie continue des groupes localement compacts. Ann. Sci. École Norm. Sup. (4), 12(2):137-168, 1979.

[BR18a] Uri Bader and Christian Rosendal. Coarse equivalence and topological couplings of locally compact groups. Geom. Dedicata, 196:1-9, 2018.

[BR18b] Marc Bourdon and Bertrand Remy. Quasi-isometric invariance of continuous group $L^{p}$-cohomology, and first applications to vanishings. Ann. H. Lebesgue, 3, 2020.

[BCR98] Jacek Bochnak, Michel Coste and Marie-Françoise Roy. Real Algebraic Geometry. Springer-Verlag, Berlin Heidelberg, 1998. 
[CdlH16] Yves Cornulier and Pierre de la Harpe. Metric geometry of locally compact groups, volume 25 of EMS Tracts in Mathematics. European Mathematical Society (EMS), Zürich, 2016. Winner of the 2016 EMS Monograph Award.

[CLM18] Alessandro Carderi and François Le Maître. Orbit full groups for locally compact groups. Trans. Amer. Math. Soc., 370(4):2321-2349, 2018.

[Cor18] Yves de Cornulier. On the quasi-isometric classification of locally compact groups. In New directions in locally compact groups, volume 447 of London Math. Soc. Lecture Note Ser., pages 275-342. Cambridge Univ. Press, Cambridge, 2018.

[Fur99a] Alex Furman. Gromov's measure equivalence and rigidity of higher rank lattices. Ann. of Math. (2), 150(3):1059-1081, 1999.

[Fur99b] Alex Furman. Orbit equivalence rigidity. Ann. of Math. (2), 150(3):1083-1108, 1999.

[Ger64] Murray Gerstenhaber. On the Deformation of Rings and Algebras. Ann. of Math. (2), 79(3):59-103, 1964.

[Gon98] Ming-Peng Gong. Classification of nilpotent Lie algebras of dimension 7. PhD Thesis, 1998. http://hdl.handle.net/10012/245

[Gra07] Willem A. de Graaf. Classification of 6-dimensional nilpotent Lie algebras over fields of characteristic not 2. Journal of Algebra, 309(2):640-653, 2007.

[Gro84] Mikhael Gromov. Infinite groups as geometric objects. In Proceedings of the International Congress of Mathematicians, Vol. 1, 2 (Warsaw, 1983), pages 385-392. PWN, Warsaw, 1984.

[Gro93] Mikhael Gromov. Asymptotic invariants of infinite groups. In Geometric group theory, Vol. 2 (Sussex, 1991), volume 182 of London Math. Soc. Lecture Note Ser., pages 1-295. Cambridge Univ. Press, Cambridge, 1993.

[Gui80] Alain Guichardet. Cohomologie des groupes topologiques et des algèbres de Lie, volume 2 of Textes Mathématiques [Mathematical Texts]. CEDIC, Paris, 1980.

[Kec95] Alexander S. Kechris. Classical descriptive set theory, volume 156 of Graduate Texts in Mathematics. Springer-Verlag, New York, 1995.

[KKR17] Juhani Koivisto, David Kyed, and Sven Raum. Measure equivalence and coarse equivalence for unimodular locally compact groups. Groups Geom. Dyn. (to appear). arXiv:1703.08121,

[KKR18] Juhani Koivisto, David Kyed, and Sven Raum. Measure equivalence for non-unimodular groups. Transform. Groups. (to appear). arXiv:1805.09063.

[Mag08] Louis Magnin. Adjoint and trivial cohomologies of nilpotent complex Lie algebras of dimension $\leqslant 7$. Int. J. Math. Math. Sci, 2008 Article ID 805305, 12 pages

[Mal49] Anatoly Mal'cev. On a class of homogeneous spaces. Izvestiya Akad. Nauk. SSSR. Ser. Mat., 13, 1949.

[Mal51] Anatoly Mal'cev. On a class of homogeneous spaces. AMS Translation, 39, 1951.

[MS06] Nicolas Monod and Yehuda Shalom. Orbit equivalence rigidity and bounded cohomology. Ann. of Math. (2), 164(3):825-878, 2006.

[Nom54] Katsumi Nomizu. On the cohomology of compact homogeneous spaces of nilpotent Lie groups. Ann. of Math. (2), 59:531-538, 1954.

[OW80] Donald S. Ornstein and Benjamin Weiss. Ergodic theory of amenable group actions. I. The Rohlin lemma. Bull. Amer. Math. Soc. (N.S.), 2(1):161-164, 1980.

[Pan89] Pierre Pansu. Métriques de Carnot-Carathéodory et quasiisométries des espaces symétriques de rang un. Ann. of Math. (2), 129(1):1-60, 1989

[Roe03] John Roe. Lectures on coarse geometry, volume 31 of University Lecture Series. American Mathematical Society, Providence, RI, 2003.

[Sau06] Roman Sauer. Homological invariants and quasi-isometry. Geom. Funct. Anal., 16(2):476-515, 2006.

[Sha04] Yehuda Shalom. Harmonic analysis, cohomology, and the large-scale geometry of amenable groups. Acta Math., 192(2):119-185, 2004.

[SS18] Roman Sauer and Michael Schrödl. Vanishing of $\ell^{2}$-Betti numbers of locally compact groups as an invariant of coarse equivalence. Fund. Math., 243(3):301-311, 2018.

[vE55] Willem T. van Est. On the algebraic cohomology concepts in Lie groups. I,II. Nederl. Akad. Wetensch. Proc. Ser. A. $\mathbf{5 8}$ = Indag. Math., 17:225-233, 286-294, 1955. 
Thomas Gotfredsen, Department of Mathematics and Computer Science, University of Southern Denmark, Campusvej 55, DK-5230 Odense M, Denmark

Email address: thgot@imada.sdu.dk

David Kyed, Department of Mathematics and Computer Science, University of Southern Denmark, Campusvej 55, DK-5230 Odense M, Denmark

Email address: dkyed@imada.sdu.dk 